PNL-3138

\title{
Definition of Gust Model Concepts and Review of Gust Models
}

David C. Powell

James R. Connell

June 1980

Prepared for the U.S. Department of Energy under Contract DE-AC06-76RLO 1830

Pacific Northwest Laboratory Operated for the U.S. Department of Energy by Battelle Memorial Institute 
NOTICE

This report was prepared as an account of work sponsored by the United States Covernment. Neither the United States nor the Department of Energy, nor any of their employees, nor any of their contractors, subcontractors, or their employees, makes any warranty, express or implied, or assumes any legal liability or responsibility for the accuracy, completeness or usefulness of any information, apparatus. product or process disclosed, or represents that its use would not infringe privately owned rights.

The views, opinions and conclusions contained in this report are those of the contractor and do not necessarily represent those of the United States Government or the United States Department of Energy.

\author{
PACIFIC NORTHWEST LABORATORY \\ operated by \\ BATTELLE \\ for the \\ UNITED STATES DEPARTMENT OF ENERGY \\ Under Contract DE-AC06-76RLO 1830
}

\author{
Printed in the United States of America \\ Available from \\ National Technical Information Service \\ United States Department of Commerce \\ 5285 Port Royal Road \\ Springfield, Virginia 22151
}

Price: Printed Copy s

$\because$ Microfiche $\$ 3.00$

NTIS

-Pages Selling Price

001-025 $\$ 4.00$

026-050 \$4.50

051-075 \$5.25

076-100 $\$ 6.00$

$101-125 \quad \$ 6.50$

$126-150 \quad \$ 7.25$

151-175 \$8.00

176-200 $\$ 9.00$

201-225 $\$ 9.25$

226-250 \$9.50

$251-275 \quad \$ 10.75$

276-300 \$11.00 


\section{9}

DEFINITION OF GUST MODEL CONCEPTS

AND REVIEW OF GUST MODELS

David C. Powell

James R. Connell

June 1980

Prepared for

the U.S. Department of Energy

under Contract DE-ACO6-75RLO 1830

Pacific Northwest Laboratory

Richland, Washington 99352 


\section{ACKNOWLEDGMENTS}

The authors gratefully acknowledge the very helpful reviews of the first draft of this document by R. E. Akins, M. A. Bowes, W. C. Cliff, J. C. Doran, G. H. Ficht1, C. H. Huang, J. V. Ramsdel1, T. R. Richards, and D. A. Spera. 



\section{SUMMARY}

This report examines four models that attempt to describe wind fluctuations in relation to a wind energy conversion system (WECS) that is subjected to these fluctuations observed from a fixed location within the atmospheric boundary layer. The primary purpose of this examination is to provide a basis for understanding present and future developments in gust and gust-rise models. The examination is accomplished by identifying the gust definitions used in the models and relating them to a basic definition given in this report.

After briefly introducing the models considered, we present a technical gust definition and some fundamental gust relations that form a basis for the review of the four models. The essentials of the models are given in a common notation to facilitate comparison. Then all of the foregoing items are used to make a critical and interpretive comparison of the four models. This is followed by some recommendations for future gust modeling.

The four models reviewed are the Ramsdell model, the Cliff-Fichtl model, the MOD-2 wind turbine model (also known as the NASA-Lewis model), and the Huang-Ficht1 mode1. There are reservations concerning each of them. The only one recommended relatively intact is the Huang-Fichtl model, which is also recommended to supercede the $\mathrm{Cliff-Fichtl} \mathrm{model.} \mathrm{We} \mathrm{recommend} \mathrm{that} \mathrm{any-}$ one wanting to use the form of the Ramsdell model reparameterize it using specific site data. We recommend the turbulence model and the mean wind probability model in the MOD-2 model and suggest repair of a logical error that occurs when turbulence and gust concepts are related. Also this model is in need of a satisfactory relation between gust amplitude and time that is a function of filtering.

Our conclusion from this examination is principally that because agreement on the appropriate gust descriptor is lacking, no one gust model can be recommended at this time. However, one basic gust model form -- the exceedance form due to Rice (1945) -- is recommended. Three species of this model are derived using the best ideas that we have found in the review of models 
plus some of our own work, which is described in Appendix B. We prefer to call these three species turbulence model, discrete gust model, and velocity-difference (gust-rise) model. The first model relies totally on conventional turbulence analysis. The second imposes a discrete gust definition on turbulence time series, wherein both gust amplitude and gust time are indigenous gust event properties. The third is a velocity-difference (gust-rise) model in which differenced data are analyzed. After these models are set forth, the strengths and weaknesses of each in principle are pointed out. However, this evaluation does not clearly indicate that any one model is superior.

The discrete gust concept set forth in Section 2 is capable of greater development than is found in any of the reviewed models. In Appendix $B$, we present a preliminary analysis that is expected to develop into a discrete gust model. This will model the statistics of discrete events that can be defined from a turbulence time series, each of which is assigned an indigenous measure of velocity amplitude and time duration.

In Appendix B, statistics of gust amplitude and gust duration are presented for the same original turbulence time series as it emerges from several digital filters, each representing a different assumed frequency range of machine sensitivity. The gust statistics, particularly those of gust duration, are strongly a function of the chosen filtering. 


\section{CONTENTS}

ACKNOWLEDGMENTS. . . . . . . . . . . . . . . . . . . . . . . . i ii

SUMMARY. . . . . . . . . . . . . . . . . . . . . . . V v

1.0 INTRODUCTION. . . . . . . . . . . . . . . . . . . . . . . . 1- 1

2.0 DEFINITIONS AND RELATIONS . . . . . . . . . . . . . . . . . 2-1

2.1 DISCRETE GUST DEFINITION . . . . . . . . . . . . . . 2-1

2.1.1 Two Treatments of Gust Time . . . . . . . . . . 2-2

2.1.2 Distinction Between Discrete Gust Model and
Turbulence Model. . . . . . . . . . . . 2-3

2.2 FUNDAMENTAL GUST-TURBULENCE RELATIONS. . . . . . . . . . . . 2-4

3.0 EXPOSITION OF GUST MODELS USING COMMON NOTATION . . . . . . . . . 3-1

3.1 THE RAMSDELL MODEL . . . . . . . . . . . . . . . . . 3-1

3.2 MOD-2 WIND TURBINE GUST MODEL. . . . . . . . . . . . . . 3-4

3.3 THE CLIFF-FICHTL MODEL . . . . . . . . . . . . . . 3-6

3.4 THE HUANG-FICHTL MODEL . . . . . . . . . . . . . . . . 3-10

4.0 COMPARISON OF GUST MODELS . . . . . . . . . . . . . . . . . 4-1

4.1 THE RAMSDELL MODEL . . . . . . . . . . . . . . . . . 4-1

4.2 THE MOD-2 WIND TURBINE GUST MODEL. . . . . . . . . . . . . . 4-11

4.3 THE CLIFF-FICHTL AND HUANG-FICHTL MODELS . . . . . . . . . . 4-14

4.3.1 Common Characteristics. ............ . 4-14

4.3.2 Comparison. .................. . . 4-18

4.3.3 Information Available from the HF Model . . . . . 4-22

5.0 RECOMMENDATIONS FOR FUTURE MODELING . . . . . . . . . . . . . . . 5-1

5.1 FOR FUTURE TURBULENCE MODELING . . . . . . . . . . . . . 5-1

5.2 FOR FUTURE DISCRETE GUST MODELING, INCLUDING VELOCITY-
DIFFERENCE MODEL . . . . . . . . . . . . . . . . . . . . . . . $5-2$

5.3 REGARDING STATIONARY OR MOVING FRAMES OF REFERENCE . . . . . 5-3

6.0 CONCLUSIONS . . . . . . . . . . . . . . . . 6-1

6.1 TURBULENCE MODEL . . . . . . . . . . . . . . . . . . 6-2

6.2 DISCRETE GUST MODEL FOR FILTERED DATA . . . . . . . . . . . $6-4$

6.3 VELOCITY-DIFFERENCE (GUST-RISE) MODEL. . . . . . . . . . . . 6-7

6.4 COMMENTARY ON THE ABOVE THREe MOdels . . . . . . . . . . . 6-8

6.5 COMMENTARY ON DISCRETE GUST MODELING AS AN IDEA. . . . . . 6-9

7.0 REFERENCES. . . . . . . . . . . . . . . . . . . . . . 7-1 
8.0 GlOSSARY. . . . . . . . . . . . . . . . . . . . . . 8-1

APPENDIX A: WIND GUST ANALYSIS FOR WIND TURBINE DESIGN. . . . . . . . A-1

APPENDIX B: PRELIMINARY DEMONSTRATION OF A DISCRETE GUST MODEL

INVOLVING INTRINSIC GUST AMPLITUDE AND INTRINS IC

GUST TIME ......................... B- . .

APPENDIX C: CALCULATION OF FREQUENCY OF ZERO-CROSSINGS FROM

TURBULENCE SPECTRA. ................ $\mathrm{C}-1$ 


\section{LIST OF FIGURES}

2.1 Two Gust Definitions ............... 2-3

4.1 Dependence of Basic Frequency Factor on Atmospheric and Machine Parameters. . . . . . . . . . 4-5

4.2a Comparison of Observed and Modeled Gust Probability for Ramsdell Gust Analysis Using No Filter. . . . . . . 4-8

4.2b Comparison of Observed and Modeled Gust Probability for Ramsdell Gust Analysis Using 50/5 sec Bandpass

Filter................. . . 4-9

4.3 Comparison of Original and Suggested Gust

Probability Density. ............ . 4-15

4.4 $\Delta u(t, \tau)$ from Nonmonotonic Change in $u(t) \ldots$. . . . . . 4-16

4.5 Discrete Events Defined for Cliff-Fichtl and Huang-Fichtl Models. . . . . . . . . . . 4-19

4.6 $N_{0}(\tau)$ in Huang-Fichtl and Cliff-Fichtl Models...... 4-21

6.1 GUST Definition .............. 6-5

B.1 Velocity Fluctuations Relative to GUST, Tolerance. . . . B B-2

B.2 Statistics for GUST Speed - No Filter . . . . . . B-8

B.3 Statistics for GUST, Speed - $50 / 5$ Bandpass Filter. . . . . B-9

B.4 Statistics for GUST, Speed With $50 / 5$ Second Bandpass Filter. . . . . . . . . . . B-12

B.5 Characteristic Magnitude Analysis for Horizontal Wind Speed .............. B-15 


\section{$\underline{\text { LIST OF TABLES }}$}

1.1 Gust Model Literature Reviewed. . . . . . . . . . .

3.1 Turbulence Intensity $k$ for Various Terrain Types. . . . . 3-2

3.2 Exceedance Parameters for Seattle Turbulence Data . . . 3-3

4.1 Summary of Characteristics of Four Gust Models. . . . . . 4-2

B.1 GUST Parameters and Ratios Among Scale Parameters from Analysis of One Test.............. 


\subsection{INTRODUCTION}

Within the past two years Pacific Northwest Laboratory (PNL) has been associated with several documents in which the authors set forth their concepts of a wind component gust model. These documents either originated at PNL or were evaluated in memoranda originating at PNL. They are listed in Table 1.1.

\section{TABLE 1.1. Gust Model Literature Reviewed}

\begin{tabular}{|c|c|c|}
\hline Abbreviation & Author & Title \\
\hline$R$ & J. V. Ramsdell & $\begin{array}{l}\text { Estimates of the Number of Large } \\
\text { Amplitude Gusts--PNL-2508, March } \\
1978\end{array}$ \\
\hline$C F$ & $\begin{array}{l}\text { W. C. Cliff } \\
\text { G. H. Fichtl }\end{array}$ & $\begin{array}{l}\text { Wind Velocity-Change (Gust Rise) } \\
\text { Criteria for Wind Turbine Design-- } \\
\text { PNL-2526, July } 1978\end{array}$ \\
\hline M2 & See Footnote (a) & $\begin{array}{l}\text { MOD-2 Wind Turbine Gust Model-- } \\
\text { published as Appendix A, this } \\
\text { report }\end{array}$ \\
\hline$H F$ & $\begin{array}{l}\text { C. H. Huang } \\
\text { G. H. Fichtl }\end{array}$ & $\begin{array}{l}\text { Gust-Rise Exceedance Statistics } \\
\text { for Wind Turbine Design--PNL-2530, } \\
\text { July } 1979\end{array}$ \\
\hline
\end{tabular}

(a) The MOD-2 Wind Turbine Gust Model is often referred to as the NASA/ Lewis gust model; see Powel1 (1979). It was originally known to us in the form of a memorandum by D. A. Spera and T. R. Richards of NASA. However, the model was actually developed principaliy by David Merchant of Boeing in consultation with W. C. Cliff of PNL and Walter Frost of FWG, Inc. The title MOD-2 Wind Turbine Gust Model has been suggested.

All of the models describe wind fluctuation statistics in a manner that can be related to detrimental effects on a WECS machine exposed to the wind at a fixed location. However, from one document to another, the authors begin by defining different discrete events as gusts and finish by formulating different parameters. Some of the differences result from the 
different purposes of the models. For example, the $R$ model is designed to predict frequency of occurrence of large gusts for the user who requires a minimum of mathematical sophistication. The HF and CF models are intended for predicting the frequency of occurrence of large gust rise events. The M2 model is intended as a fatigue model. Because of these differences, therefore, the documents lack a basis for comparative discussion.

This review seeks to provide a framework for understanding these models and a basis for future communication. We hope to correct the present situation in which the reader considering all of the referenced documents may find that ideas have been multiply defined and inadequately related.

This report begins with an original definition of discrete gust that is related to established concepts of atmospheric turbulence and differentiated from them. In developing our definition, we have two basic options; "gust" may either be taken as synonymous with turbulence or as a concept that is not defined in conventional turbulence literature. We have chosen the latter option. Following the discrete gust definition and illustration, fundamental gust relations are set forth that provide the basis of the remainder of the review. 


\subsection{DEFINITIONS AND RELATIONS}

No technical definition of gust is uniformly accepted within the community of design engineers and atmospheric scientists involved with gust model literature. A11 of the gust model documents reviewed in this report define discrete events in terms of some aspect of the process called turbulence. The $\mathrm{H} 2$ memorandum most strongly implies some philosophic difference by using the phrase "discrete gusts".

This section presents an original definition of a discrete gust, and identifies a set of relations between discrete gusts and turbulence. In following sections, the behavior of the models reviewed is discussed with respect to our definition.

\subsection{DISCRETE GUST DEFINITION}

It is possible to encompass all the separate variations on the gust concept found in these documents in one definition of discrete gusts. This definition applies to a foreign body exposed to the atmosphere at a fixed location, where it experiences the gust phenomena as events in time, such as an anemometer that records the passage of wind fluctuations as a time series. Within this framework we define gusts as constituting any series of discrete velocity-time events that can be defined from a turbulence time series according to some extrinsic criterion.

Three comments should be made about this definition before proceeding. First, this is an operational definition. The extrinsic criterion depends on some notion of the responsiveness of a foreign body subjected to atmospheric gusts. This is an application that is extrinsic to the consideration of atmospheric phenomena per se. Second, this definition does not imply that discrete gusts are intermittent patches of turbulence distinguished by large fluctuations. However, the extrinsic criterion could define a minimum magnitude for wind fluctuations that qualify as gusts. In this particular case, the discrete gust definition would correspond more closely to the physical imagery the word conveys. Third, since each discrete gust event has two tags, velocity and time, there must be both a gust velocity scale and a gust 
time scale. It remains to be seen whether or not these can be derived from corresponding velocity and time scales of turbulence that are already wellknown by definition, and for basic atmospheric states that are well quantified in the existing literature.

\subsubsection{Two Treatments of Gust Time}

There are two fundamentally different treatments of gust time (also called gust period or duration by some writers). In some models the gust time is arbitrarily fixed; in other models the gust time varies. The relevance of gust time over a fixed interval has been stated by Kerrigan (1977): "An estimate of the frequency with which this wind component changes by a given amount in a given time is regarded as important." Mathematically, Kerrigan's model reduces to defining statistics of velocity change for an arbitrarily fixed time interval. This is also the root of the later treatments in the Cliff-Fichtl and Huang-Fichtl models. Frost et al. (1978) state in the section of their handbook on discrete gust modeling: "The prediction of gust magnitude given in this report assumes that the duration, $\tau$, of the required design gust is a known parameter determined from the dynamic analysis of the WTG." Again the implication is that the gust time is fixed.

The other treatment allows gust time to vary from one gust to another. This treatment is implicit in the M2 model memorandum in that the authors speak of the most probable time $T$ of a gust with amplitude $A$. Each of the gust events defined by Ramsdell also has an individual time duration.

Two examples of this latter type of gust definition, shown in Figure 2.1, represent a sample of wind fluctuation in the form of a windcomponent time series. The discrete events are delimited by adjacent crossings of the zero value in the first series; i.e., the GUST definition. The discrete events are delimited by adjacent zeros of the first derivative in the second series; i.e., the GUST 1 definition. In each case, the pair $\left(A_{i}, T_{i}\right)$ illustrates the required measures of velocity and time increment.

Finally, we observe that our discrete gust definition is complementary to standard turbulence definitions. These apply to a homogeneous phenomenon 



FIGURE 2.1. Two Gust Definitions

that is represented in analysis by stationary time series. However, the standard treatment does not recognize the importance of analysis of measures of discrete events that may be defined within the time series. In the final statistical analysis of turbulence the significance of discrete events is 10st. Therefore, another way to phrase our discrete gust definition is to say that gusts consist of arbitrarily defined discrete events that can be defined from turbulent flow. For our purpose, such a definition is related to notions of machine responsiveness to wind fluctuations.

\subsubsection{Distinction Between Discrete Gust Model and Turbulence Model}

We propose that a discrete gust model be one from which the statistics are those of discrete events defined using an arbitrary criterion that cannot be derived from standard turbulence definitions. In contrast, a turbulence model is one from which the statistics are those that can be derived using accepted turbulence definitions. 


\subsection{FUNDAMENTAL GUST-TURBULENCE RELATIONS}

From the definition of discrete gusts of Section 2.1 and from the requirements of application, it follows that we are interested in joint probability descriptions of gust velocity and gust times. Also, we are interested in the frequency of occurrence of gust events of certain magnitude classes. (a)

The basic structure of a discrete gust model can be expressed by mathematical formalism developed from the following specifications:

- one basic state of the atmosphere $S$, which depends on mean wind speed $U$, thermal stability, surface roughness $z_{0}$, and which is a function of height above the surface $z$

- one definition of discrete events $E$

- a subset of events $E$ that can be characterized by the magnitude classes $x$ and $y$ for gust amplitude and gust time, respectively

- a turbulence spectral function $\phi(n)$, where $n$ is cyclical frequency. Although $\phi(n)$ is a function of $S$, we shall surpress the $S$ notation.

- one machine filter function $F(n)$.

Now we can write the basic mathematical structure of a discrete gust model as follows:

$$
\left[N_{A T}(x, y ; S, F)=N_{0}(S, F) P_{A T}\left(x, y \mid \sigma_{A}, \sigma_{T}, \rho_{A T}, S, F\right]_{E}\right.
$$

(a) Throughout this review the descriptors "probability" and "frequency of occurrence" are associated with the following type of notation:

$$
P_{u}(x) \text { or } N_{u}(x) \text {. }
$$

The first of these is a probability and the second a frequency of occurrence. The notation is that used in random variable analys is. In either case the expression concerns the random variable $u$ when $u$ is in a subset $x$. The first expression is the probability of encountering values of $u$ that are greater than $x$. The second is the frequency of occurrence of values of $u$ that are greater than $x$. If the second notation is in the context of level crossings, it designates the frequency of positive slope level crossings of the value $x$ by the random variable $u$. 


$$
\begin{gathered}
\left(\sigma_{A}\right)_{E}=\left(\sigma_{A}\right)_{E}[\sigma(S, F), S, F, E] \\
{\left[\sigma_{T}=\sigma_{T}\left[N_{0}(S, F), S, F\right]\right]_{E}} \\
\left(\rho_{A T}\right)=\left(\rho_{A T}\right)[S, F, E] \\
\sigma^{2}(S)=\int_{0}^{\infty} \phi(n) d n \\
\sigma^{2}(S, F)=\int_{0}^{\infty} \phi(n) F(n) d n .
\end{gathered}
$$

$N_{A T}(x, y ; S, F)$ is the frequency of occurrence of events where simultaneously the gust amplitude $A$ exceeds $x$ and the gust time $T$ exceeds $y$. The $S$ and $F$ notations indicate dependence on the state of the atmosphere and on the assumed filtering properties of the machine. $N_{0}(S, F)$ is the basic frequency-of-occurrence factor, which will be called the basic frequency factor hereafter. The physical significance of the basic frequency factor, which varies from one model to another, will be discussed later. Here we simply point out that $N_{0}(S, F)$ is the factor on the right that gives the term on the left its dimension, which is that of inverse time. $P_{A T}(x, y)$ is a dimensionless probability factor that expresses the probability that for a given gust event $A$ exceeds $x$ and $T$ exceeds $y$, simultaneously.

The information in the joint probability description can also be used to generate the conditional probability of amplitude when time is given, the conditional probability of time when amplitude is given, and the marginal probabilities of amplitude and of time, respectively.

If, for a given discrete definition $E$, the model of Equation (2.1) is to be a practical model, the probability structure must be amenable to description, and the dependencies on $S$ and $F$ indicated at the extreme right of Equations (2.1a) through (2.1d) must be tractable. 
A special (and simplified) case of the gust relations exists when the discrete event definition is such that all discrete events have the same arbitrarily imposed time duration, such as in the gust-rise or velocitydifference models (CF and HF models, respectively).

$$
\begin{gathered}
{\left[N_{A}(x ; S, F)=N_{0}(S, F) P_{A}\left(x \mid \sigma_{A}\right), S, F\right]_{E(T)}} \\
\left(\sigma_{A}\right)_{E(T)}=\left(\sigma_{A}\right)_{E(T)}[\sigma(S, F), S, F, E(T)] \\
\sigma^{2}(S)=\int_{0}^{\infty} \phi(n) d n \\
\sigma^{2}(S, F)=\int_{0}^{\infty} \phi(n) F(n) d n .
\end{gathered}
$$

In this case the probability factor may be an exceedance probability as in the CF model; if the discrete events are level crossings, as in the HF model, the probability factor, while remaining dimensionless, has otherwise the structure of probability density.

If the model of Equation (2.2) is used to calculate fatigue, the mean wind speed must figure in the argument list on the left. In practice, mean wind speed is often synonymous with the state of the atmosphere S. If the model is used to calculate the frequency of events over a long period of time, containing a variety of atmospheric conditions, one further equation must be supplied:

$$
\left[N_{A}(x ; F)=\int_{S} N_{A}(x, S, F) p(S) d S\right]_{E(T)}
$$

which illustrates, in principle, integration over all states of the atmosphere, where $p(S)$ is a probability density such that 


$$
\int_{0}^{\infty} p(S) d S=1 .
$$

It should be noted that Equation (2.3) implies that no matter how rare the event is, it is part of an atmospheric condition that we can describe as a stationary process. That such a rare event (occurring perhaps only once over the years that a WECS is projected to operate) would occur in a steadystate process that $p(S)$ describes is very doubtful.

In the special case (see CF and HF models) where the arbitrary discrete event's time is imposed by velocity data that have been differenced at that time interval (see Equation (3.3)), Equation (2.2b) may be written

$$
\left(\sigma_{A}\right)_{E(\tau)}^{2}=\int_{0}^{\infty} \phi(n, \tau) F(n) d n
$$

(see Equations (3.19) and (3.20)).

It turns out that in Equations (2.2), (2.3), and (2.5) the generalized amplitude symbol $A$ is identical to the velocity difference $\Delta u$ defined by Equation (3.3). In subsequent parts of the report where the $A$ is used, it represents a discrete gust amplitude of the GUST, or GUST, type (except for usage in Sections 3.2 and 4.2 in connection with the M2 model). 


\subsection{EXPOSITION OF GUST MODELS USING COMMON NOTATION}

In this section the essential equations of the four gust models are given in a common notation that facilitates comparison. We have omitted equations dealing with vertical extrapolation of mean wind speed.

We begin with the customary turbulence definition, which recognizes a direction of mean flow and divides the total of the component of flow in that direction into fluctuating and mean parts according to

$$
U(t)=u(t)+U
$$

where

$$
U=\frac{1}{T_{s}} \int_{0}^{T_{s}} U(t) d t
$$

and where $T_{S}$ is an averaging time on the order of 10 minutes to an hour over which wind conditions approach stationarity.

Some models use the term "gust rise" to refer to velocity change over a set period of time $\tau$. Therefore, we define the velocity change descriptor as

$$
\Delta u(t, \tau)=u(t)-u(t+\tau) .
$$

A11 references to $\Delta u$ in this report, subscripted or not, refer to this velocity change.

\subsection{THE RAMSDELL MODEL}

The discrete events of the $R$ model are identical with the GUST 0 events shown in Figure 2.1. However, each event is tagged with positive or negative amplitude. There is no time tag. The model is more empirical than the others and is designed for users who require minimum sophistication. Although Ramsdell only discussed the u-component, the model can be used 
equally well for the $v$ - and $w$-components if they are appropriately

parameterized.

Let $N_{A}(x ; U)$ be the frequency of encountering $u$-component GUST amplitudes $A$ that exceed the value $x$ when the mean wind speed is $U$. (See also the remarks on notation in Section 2.2.) This is given by

$$
N_{A}(x ; U)=N_{u}(0) \exp \left[-a\left(x / \sigma_{u}\right)^{b}\right]
$$

where $N_{u}(0)$ is the frequency of zero-crossings with positive slope, $\sigma_{u}$ is the root mean square ( $r m s$ ) of u-component turbulence and a and b are empirically determined constants. $\sigma_{u}$ is related to the mean wind speed by

$$
\sigma_{u}=k U
$$

where $k$ is one of the turbulence intensity values for terrain types given in Table 3.1 .

TABLE 3.1. Turbulence Intensity $k$ for Various Terrain Types

\begin{tabular}{ll}
\multicolumn{1}{c}{ Terrain Type } & $k$ \\
\hline smooth/open water & 0.10 \\
flat/gently rolling & 0.15 \\
hilly & 0.20 \\
low mountains & 0.25 \\
high mountains & 0.30
\end{tabular}

The $U$ dependence comes from $\sigma_{u}$ as defined by Equation (3.5) and Table 3.1.

Ramsde11 points out that steady-state loads on a WECS are a function of mean wind speed. Therefore, the final descriptor $N_{A}(x ; U)$ must be a function of both gustiness $x$ and mean wind speed $U$. 
Ramsde11 also gives an equation for the number of gusts per year of amplitude $A$ exceeding $x$ when the mean wind speed is between $U_{1}$ and $U_{2}$. This can be written

$$
Y_{A}\left(x ; U_{1}, U_{2}\right)=8766 N_{u}(0) e^{-a\left(\frac{x}{\sigma_{u}}\right)^{b}} P\left\{U_{1} \leq U \leq U_{2}\right\}
$$

where

$$
P\left\{U_{1} \leq U \leq U_{2}\right\}=e^{-\frac{\pi}{4}\left(\frac{U_{1}}{U_{a}}\right)^{2}}-e^{-\frac{\pi}{4}\left(\frac{U_{2}}{U_{a}}\right)^{2}}
$$

is a Rayleigh distribution with $U_{a}$ as the annual average mean wind speed. In Equation (3.6) $\mathrm{N}_{u}(0)$ must be the number of positive-slope zero crossings per hour since 8766 is the number of hours per year.

The parameterization of this model results from an analysis of many data segments from several heights above the surface that involve three complex terrain locations in the Seattle area. Ramsdell states that a value of

$$
N_{u}(0)=1150 \mathrm{hr}^{-1}
$$

holds reasonably well at all heights and mean wind speeds. The constants $a$ and $b$ have slightly different values for positive and negative gusts. They are given in Table 3.2 .

TABLE 3.2. Exceedance Parameters for Seattle Turbulence Data

\begin{tabular}{lll} 
& $\mathrm{a}$ & $\mathrm{b}$ \\
\cline { 2 - 3 } Positive Gusts & 1.03 & 1.43 \\
Negative Gusts & 1.16 & 1.53
\end{tabular}


The document also has an appendix of extensive gust exceedance tables for various mean wind classes and terrain types. As explained in Section 4, the numerical specifications quoted above are invalid.

\subsection{MOD-2 WIND TURBINE GUST MODEL}

This model is known to us through a previously unpublished memorandum, which is attached to this report as Appendix A. Since the equations are given there, only those pertinent to the scope of this report are given here in uniform notation, with the briefest of comment.

This model differs from the other three in this chapter in that the final calculation is of dimensional probability rather than of frequency of occurrence. These data are subsequently used to calculate machine fatigue. In addition, this is the only model that uses the phrase discrete gust.

The mean wind model is that of Weibull probability distribution. If $U_{p}$ is a particular mean wind speed, the probability that a mean wind speed $U$ exceeds $U_{p}$ is given by

$$
\left[P\left\{U \geq U_{p}\right\}=e^{-\left(U_{p} / c\right)^{k}}\right]_{\text {ref }}
$$

where ref refers to a reference level of $9.1 \mathrm{~m}$ and $c$ and $k$ are empirically determined dimensional and dimensionless constants, respectively.

The relation of the u-component turbulence rms to the mean wind is given by

$$
\left[\sigma_{u}=\frac{1.04 U}{\operatorname{Ln}\left(1+\frac{z}{z_{0}}\right)}\right]_{\text {ref }} \text {. }
$$


In order to compute the final probability density, the $M 2$ model uses the rms value of filtered turbulence. This is the integral of the turbulence spectrum between upper and lower frequency limits of sensitivity of machine response. This rms can be written

$$
\sigma_{u}(F)=\left[\int_{n_{1}}^{n_{2}} n \phi_{u}(n) d[\ln (n)]\right]^{1 / 2}
$$

where the frequencies $n_{1}$ and $n_{2}$ represent the frequency 1 imits of machine sensitivity and where the $F$ notation indicates that $\sigma_{u}$ is for arbitrarily filtered turbulence. In this equation, $F$ is specified by the limits of integration. $n \phi_{u}(n)$, the spectrum of $u$-component turbulence, is given by

$$
\frac{n \phi_{u}(n)}{\left[\sigma_{u}^{2}\right]_{\text {ref }}}=\frac{0.164\left(f / f_{o u}\right)}{1+0.164\left(f / f_{o u}\right)^{5 / 3}}
$$

where

$$
\begin{aligned}
f & =n z / U(z) \\
f_{\text {ou }} & =0.0144
\end{aligned}
$$

and where $z$ is probability distinct from the reference level. Equations (3.12) and (3.13) are given by Kaima 1 (1973) for stable conditions. Frost et a). (1978) supplied Equation (3.14) to adapt the spectrum to neutral conditions. The $M 2$ mode 1 also presents formulae for the turbulence rms and specific values of $f_{0}$ for the lateral and vertical turbulence components. $A$ set of unique power law equations relating mean wind speed at level $z$ to mean wind speed at a reference level is also given.

To facilitate our discussion of this model in Section 4 , we change the order of the next two equations. We introduce a quantity $A$ by the probability density expression 


$$
p(A ; U, F)=\frac{1}{\sqrt{2 \pi} \sigma_{u}(F)} e^{-\frac{1}{2}\left[\frac{A}{\sigma_{u}(F)}\right]^{2}} .
$$

The $U$ dependence notation is added on the left to indicate that this probability is at a specific value of $U$. The $U$ dependence on the right is in $\sigma_{u}(F)$; the notation is suppressed because $\sigma_{u}$ cannot be otherwise than dependent on $U$. Before this equation appears in the memorandum, the same $A$ is given as a gust amplitude in an equation prefaced with the following sentences: "The turbulence of the wind is assumed to consist of a set of discrete gusts with Gaussian random amplitudes but with specified (deterministic) shapes and periods. The assumed shape of each discrete gust is:"

$$
U(t)=U+A(1-\cos (2 \pi t / T)) \quad 0 \leq t \leq T
$$

where $t$ is time, $A$ is gust amplitude, and $T$ is gust period. (Underlining in the quote is by the present authors). A may be positive or negative. The $T$ is the most probable period of a gust of amplitude $A$.

\subsection{THE CLIFF-FICHTL MODEL}

The CF and HF models may be called gust-rise or velocity-difference models; in contrast, the $\mathrm{R}$ and $\mathrm{M} 2$ models are called gust models. This is because the data for the $C F$ and $H F$ models are derived from $\Delta u(t, \tau)$ as defined in Equation (3.3) rather than from $u(t)$. The title of the CF model indicates a preference for the designation velocity-change model. We also prefer this designation since it does not inappropriately connote a bias in favor of positive velocity change.

Although both models are intended to calculate probabilities of extreme events, we find the models perhaps more appropriate for fatigue calculations when the mean wind speed is retained as an argument in the final parameter calculations. 
It turns out that the initial data for the CF model use the differencing interval for discretization as well as differencing. Thus in the CF model the wind data are:

$$
\begin{aligned}
& \Delta u_{1}=u\left(t_{0}+\tau\right)-u(t) \\
& \Delta u_{2}=u\left(t_{0}+2 \tau\right)-u\left(t_{0}+\tau\right) \\
& \Delta u_{k}=u\left(t_{0}+k \tau\right)-u\left(t_{0}+k(\tau-1)\right) .
\end{aligned}
$$

The final calculation in this model is the frequency of exceedance by these data of a given value $x$ of the variable $\Delta u$. At a given mean wind speed $U$, the frequency of exceedance is given by

$$
N_{\Delta u(\tau)}(x ; U, F)=\frac{1}{2 \tau}\left[\frac{1}{\sqrt{\pi / 2} \sigma_{\Delta u}(F)} \int_{x}^{\infty} e^{-\frac{1}{2}\left[\frac{\Delta u}{\sigma_{\Delta u}(F)}\right]^{2}} d \Delta u\right]
$$

where $\sigma_{\Delta u}(F)$ is the rms of $\Delta u$ of filtered turbulence.

Derivation of $\sigma_{\Delta u}(F)$ requires specification of a power spectrum and a filter such that

$$
\sigma_{\Delta u}^{2}(F)=\int_{0}^{\infty} \phi_{\Delta u}(n) F(n) d n
$$

It can be shown analytically that when $\Delta u(t, \tau)$ is defined as in Equation (3.3), the spectrum of this differenced series is related to the spectrum of the original by

$$
\phi_{\Delta u}(n, \tau)=2 \phi_{u}(n)(1-\cos 2 \pi n \tau) \quad .
$$


The power spectrum for the u-component used is the Dryden spectrum, which is given by

$$
\frac{n \phi_{u}(n)}{\sigma_{u}^{2}}=\frac{4 N}{1+(2 \pi N)^{2}}
$$

where $\mathrm{N}$ is a dimensionless frequency involving the mean wind $U$ and the integral length scale $L_{u}$ is the u-component turbulence according to

$$
N=n L_{u} / U
$$

The filter is a function of machine diameter $D$ and may be written

$$
F(n)=\frac{1}{1+\left(\frac{n D}{U}\right)^{2}} .
$$

Substituting the last four equations into Equation (3.19) yields

$$
\sigma_{\Delta u(\tau)}^{2}=2 \sigma_{u}^{2}\left[\frac{\left(1-e^{\left.-\frac{U \tau}{L_{u}}\right)}-\frac{D}{2 \pi L_{u}}\left(1-e^{-\frac{2 \pi U \tau}{D}}\right)\right.}{1-\frac{D^{2}}{\left(2 \pi L_{u}\right)^{2}}}\right] .
$$

$\sigma_{u}$ is related to the mean flow by

$$
\sigma_{u}=\frac{U}{\operatorname{Ln}\left(z / z_{0}\right)}
$$

The integral length scale is obtained from the integral time scale and the mean wind speed according to 


$$
L_{u}=U T_{u}
$$

where the integral time scale $T_{u}$ is theoretically the integral of the autocorrelation function:

$$
\tau_{u}=\frac{1}{\sigma_{u}^{2}} \int_{0}^{\infty} u(t) u(t+\tau) d t .
$$

The practical form of $L_{u}$ used in this model recognizes that $L_{u}$ is a function of height and roughness. The expression, derived from work by Counihan (1975), is

$$
\begin{aligned}
& L_{u}=\frac{25 z^{c}}{z_{0}^{0.4}} \\
& c=\exp \left[-0.025\left(\operatorname{Ln} z_{0}\right)^{2}+0.17 \operatorname{Ln} z_{0}-0.8\right]
\end{aligned}
$$

where three values of $z_{0}$ are offered:

$$
\begin{aligned}
& z_{0}=0.005 \mathrm{~m} \text { (smooth terrain) } \\
& z_{0}=0.05 \mathrm{~m} \text { (moderate terrain) } \\
& z_{0}=0.34 \mathrm{~m} \text { (rough terrain) }
\end{aligned}
$$

The development from Equation (3.18) to Equation (3.27) is a function of mean wind speed. In order to obtain an exceedance frequency that holds for a long period of time over which the mean wind speed varies, we must integrate over the probability density of mean wind speed:

$$
N_{\Delta U(\tau)}(x ; F)=\int_{0}^{\infty} N_{\Delta U(\tau)}(x ; U, F) p(U) d U
$$


The probability density used is the Rayleigh density:

$$
\begin{aligned}
p(U) & =\frac{U}{\theta^{2}} e^{-\frac{1}{2}\left(\frac{U}{\theta}\right)^{2}} \\
\theta & =\sqrt{\frac{2}{\pi}} U_{a}
\end{aligned}
$$

where $U_{a}$ is a climatological mean wind speed. If mean wind speeds limited to a given cutout speed $U_{c}$ are to be considered, the exceedance frequency is given by

$$
N_{\Delta u(\tau)}\left(x ; U_{a}, F\right)=\int_{0}^{U_{a}} N_{\Delta u(\tau)}(x ; U, F) p(U) d U
$$

Finally, if $T_{d}$ is the design lifetime of the machine, the probability of encountering a gust of magnitude $\Delta u=x$ in that lifetime is

$$
P_{\Delta u(\tau)}\left(x ; U_{a}, F\right)=1-\exp \left[T_{d} N_{\Delta U(\tau)}\left(x ; U_{a}, F\right)\right] \text {. }
$$

Since the model does not treat vertical extrapolation of mean wind, all of the above must be applied at level $z$, which is assumed to be hub height. Equations (3.29) and (3.31) are conveniently written in the model document to yield number of occurrences per year rather than frequency of occurrence, regardless of units, as given here.

\subsection{THE HUANG-FICHTL MODEL}

The general purposes of the HF model are identical with those of the CF model. Nevertheless, the two models employ different data, different events and different final descriptors. 
The data for this model should be regarded as a continuous function $\Delta u(t, \tau)$ as defined in Equation (3.3). When actual field data are used, $\Delta u$ will be represented by a time series involving a discretization interval. However, this interval may be as small as measuring conditions permit, and certainly should be much smaller than the differencing interval $\tau$. Therefore, the discretization of the CF model given in Equation (3.17) does not apply at a11 to the HF model, where we may consider $\Delta u$ to be a continuous function.

The discrete events of the HF model are positive-slope crossings of a level $x$ by the $\Delta u$ function. The final descriptor is the frequency of occurrence of these level crossings. The development includes the statistical theory of Rice (1945) and meteorological specifications dependent on atmospheric stability as well as on height and roughness. Logic dealing with extrapolation from a reference level to the hub height level is also worked into these equations, based on the assumption of a stability-modified logarithmic profile. However, in the section of the document illustrating the calculations for a given case, the stability treatment is omitted. Therefore, we omit stability in the exposition to follow, as well as the profile treatment, which as previously stated is not being reviewed in the present report.

When the mean wind speed is maintained as an argument, the frequency of occurrence of positive slope crossings at level $x$ by the $\Delta u$ function is given by

$$
N_{\Delta u(\tau)}\left(x ; U, n_{c}\right)=N_{\Delta u(\tau)}\left(0 ; U, n_{c}\right) e^{-\frac{1}{2}\left[\frac{x}{\sigma_{\Delta u}}\right]^{2}}
$$

where $\mathrm{N}_{\Delta u}(0)$ is the frequency of zero crossings, and is given by

$$
N_{\Delta u(\tau)}\left(0 ; U, n_{c}\right)=\left[\frac{\int_{0}^{n_{c}} n^{2} \phi_{\Delta u}(n, \tau) d n}{\int_{0}^{\infty} \phi_{\Delta u}(n, \tau) d n}\right]^{1 / 2} .
$$


The form of this expression is based on work of Rice (1945), which includes the assumption that the $\Delta u(t, \tau)$ function is Gaussian. The cutoff frequency $n_{c}$ is determined to exclude all wavelengths that are too small for the machine to be sensitive to them, i.e., shorter than $U / \mathrm{n}_{\mathrm{C}}$. The spectrum used is the same as that found in the CF model; see Equations (3.20), (3.21), and (3.22). The length scale $L_{u}$ is also the same as that used in the CF model; see Equation (3.28). However, the set of equations in the HF document labeled (28) is in error in that the left side of the last equation in the set should be $\operatorname{Ln}(1 / n)$ rather than $1 / n$.

For neutral conditions the relation between $\sigma_{u}$ and $U$ is that of Equation (3.25). The relation between $\sigma_{u}$ and $\sigma_{\Delta u(\tau)}$ is that which is obtained from Equation (3.24) when the machine diameter is set at zero. The expression is

$$
\sigma_{\Delta u}^{2}=2 \sigma_{u}^{2}\left(1-e^{-U \tau / L} u\right)
$$

The frequency of level crossings for long periods of time over which $U$ varies is developed from the frequency of level crossings at a specific $U$ as is done in the CF model, with the cutoff frequency $n_{C}$ substituting for the filter notation $F$ in Equations (3.29), (3.30), and (3.31). Thus,

$$
N_{\Delta u(\tau)}\left(x ; U_{a}, n_{c}\right)=\int_{0}^{U_{C}} N_{\Delta u(\tau)}\left(x ; U, n_{c}\right) p(U) d U
$$

where $p(U)$, the probability density of mean wind, is given by Equation (3.30). Also, if $T_{d}$ is the lifetime of a machine, the probability of encountering a level crossing at level $x$ by the $\Delta u(t, \tau)$ process is given by

$$
P_{\Delta u(\tau)}\left(x ; U_{a}, n_{c}\right)=1-\exp \left[T_{d} N_{\Delta u}\left(x ; U_{a}, n_{c}\right)\right] \text {. }
$$




\subsection{COMPARISON OF GUST MODELS}

In this interpretive section, we analyze the equations and descriptions of the four gust models given in Section 3 in relationship to the theoretical framework developed in Section 2. The analysis roughly follows the order of the key characteristics of the models identified in Table 4.1. Because the $\mathrm{Cl}$ iff-Fichtl and Huang-Fichtl models are similar in form, they are discussed in the same subsection.

\subsection{THE RAMSDELL MODEL}

As shown in Equation (3.4), the $R$ model predicts frequency of occurrence of GUST 0 amplitudes. In our analysis, we interpret the $R$ model with respect to Equation (2.1a) in which the frequencies of both GUST 0 amplitude and time are considered. Therefore, a version of Equation (2.1a) that fits this model is (for the u-component)

$$
\left[N_{A}(x ; S)=N_{0}\right]_{G U S T_{0}} P_{A}\left(x \mid \sigma_{U}(S)\right)
$$

where the term on the left is the frequency of occurrence for GUST 0 amplitudes with absolute value exceeding $x, N_{0}$ is the generalized notation for basic frequency factor, and $P_{A}\left(x / \sigma_{U}(S)\right)$ is the probability factor relating the frequency of occurrence of GUST $_{0}$ amplitudes $A$ exceeding $x$ to the basic frequency factor $N_{0}$. For GUST 0 analys is of the $u$-component of turbulence (and for the R model) $N_{0}$ is $N_{u}(0)$, the frequency of occurrence of positiveslope zero crossings by the u-component. For the $\mathrm{R}$ model in particular, the probability factor is $\exp \left[-a x / \sigma_{u}\right]^{b}$, where $a$ and $b$ are to be determined experimentally. Note that $\sigma_{u}$, the turbulence rms, rather than $\sigma_{A}$, the GUST $_{0}$ rms, is used. However, according to Equation (2.1b) these sigmas should be related. There is no reference to the filter function $F(n)$ in Equation (4.1). Ramsdell acknowledges the importance of machine filtering but does not develop the idea in the equations. 
TABLE 4.1. Summary of Characteristics of Four Gust Models

Descriptor of Wind Fluctuations

Discrete event amplitude definition

Discrete event time definition

Basic frequency factor

Assumed probability distribution of event

magnitudes

$\stackrel{p}{i}$

\section{GUST, events}

Implicitly that of GUST events

Frequency of positiveslope zero crossings (R)

Exponential

None

Rayleigh-distributed mean wind

None

None

One-minus-cosine waves
of specified amplitude
"Most probable time" of
gust with specified
amplitude (R)

amplitude (R)

Not documented, understood to be blade rota tion rate

Gaussian

Modified Kaimal-stable

(R)

Weibull-distributed mean wind

NASA power law

Sharp-cutoff band representing range of fre quency sensitivity of blade
Huang-Fichtl

Positive-slope level crossings of a time series differenced at interval $\tau$

Arbitrarily fixed by analyst

Frequency of positive level slope zero crossings ( $R$ )

Gaussian

Dryden (NR)

Rayleigh-distributed mean wind

Log-linear $(R)$

Function of blade rotation disk diameter gradual high frequency cutoff
Cliff-fichtl

Successive velocity differences at ends of

fixed time interval

Arbitrarily fixed by analyst

$0.5 \tau(N R)$

Gaussian

Dryden (NR)

Rayleigh-distributed mean wind

$\log (R)$

Function of blade rotation disk diameter gradual high frequency cutoff 
The form of the model and its parameterization are entirely empirical. Both were conceived from interpretation of the output of a computer subroutine that was part of a larger analys is program written by Powell within the period 1968-1973. Unfortunately this particular subroutine was in error such that calculated frequencies of occurrence were inflated. The true calculated values are not recoverable unless the original data are reanalyzed with a properly written code.

The data sample used in development of the Ramsde11 model (Ramsde11 1975) was voluminous. It covered about 50 periods distributed among three sites in Seattle, Washington (Ramsde11 1975): one at a lake shore, one at an airport, and one atop an antenna over a tall building in downtown Seattle. The parameterization of the $R$ model resulted from the combined analysis of data from a 11 three sites with the aforementioned computer code. Significant differences were not found by Ramsdell when the data were separated by site.

In 1978 and 1979 a new computer code was written specifically to analyze turbulence time series as GUST $_{0}$ and GUST $_{1}$ events. This code was used, with and without the defective subroutine, to reanalyze the data from the Hanford vertical plane array used for the analys is in Appendix $B$. When these data were analyzed without application of a digital filter, it was found that the ratio of the actual frequency of positive-slope zero crossings to the figure calculated using the defective subroutine was 0.36 . When the digital filters (described in Appendix B) were applied to simulate a bandpass filter (consisting of a 50-second highpass filter and a 5-second lowpass filter, called a $50 / 5 \mathrm{sec}$ bandpass filter hereafter), the ratio of the correct figure to the erroneous one was 0.47 . The first of these anaiyses, that without the digital filter, represents the conditions under which Ramsdell analyzed his data. Therefore if a correct count of positive-slope zero crossings had been employed, we estimate that his frequency of positive-slope zero crossings averaged over all analyses would have ranged from 400 to $450 \mathrm{hr}^{-1}$ rather than $1150 \mathrm{hr}^{-1}$. 
Before presenting more data analysis we should consider the existing theory that is pertinent to the structure of the Ramsdell model. An expression for the frequency of positive-slope zero crossings was developed by Rice (1945). When we incorporate machine filtering, $F(n)$, the frequency of occurrence for positive-slope zero crossings in the turbulence seen by a machine becomes:

$$
N_{U}(0, S, F)=\left[\frac{\int_{0}^{\infty} n^{2} \phi_{u}(n ; S) F(n) d n}{\int_{0}^{\infty} \phi_{u}(n ; S) F(n) d n}\right]^{1 / 2} .
$$

$\mathrm{N}_{u}(0 ; S, F)$ is the frequency of positive-slope zero crossings in the $u$-component turbulence, where the average value of $u$ is zero. (0ther notation was defined in Section 2.2.) The theory assumes that the function with spectrum $\phi_{u}(n) F(n)$, the u-component turbulence as seen by the machine, is Gaussian.

In Appendix $C$ this equation is again presented and adapted into a form suitable for calculation using a digital computer or hand calculator. The filter is reduced to a pair of cutoff frequencies (see Equation ( $C-1)$ ). Calculations produced using this formulation, with the recommended turbulence spectrum, would be theoretically appropriate for a level, homogeneous site such as a Kansas wheat field.

To express the filter in terms of cutoff frequencies $n_{1}$ and $n_{2}$, we changed the notation to $\mathrm{N}_{u}\left(0 ; S, n_{2}: n_{1}\right)$. According to Equation $(C .4)$, this quantity should vary systematically as a function of $U, z, n_{2}$ and $n_{1}$. ( $U$ and $z$ are both represented in the $S$ notation in the arguments of $N_{u}(0)$; they are present in the $f$ in Equation (C.4) since $f=n z / U$. )

The theoretical dependence of $N_{u}(0)$ on these four variables, subject to a selection of original data, is shown in Figure 4.1. The three lines show the variation of $N_{u}(0)$ as a function of $n_{2}, U$ and $z$; the heavy dots 


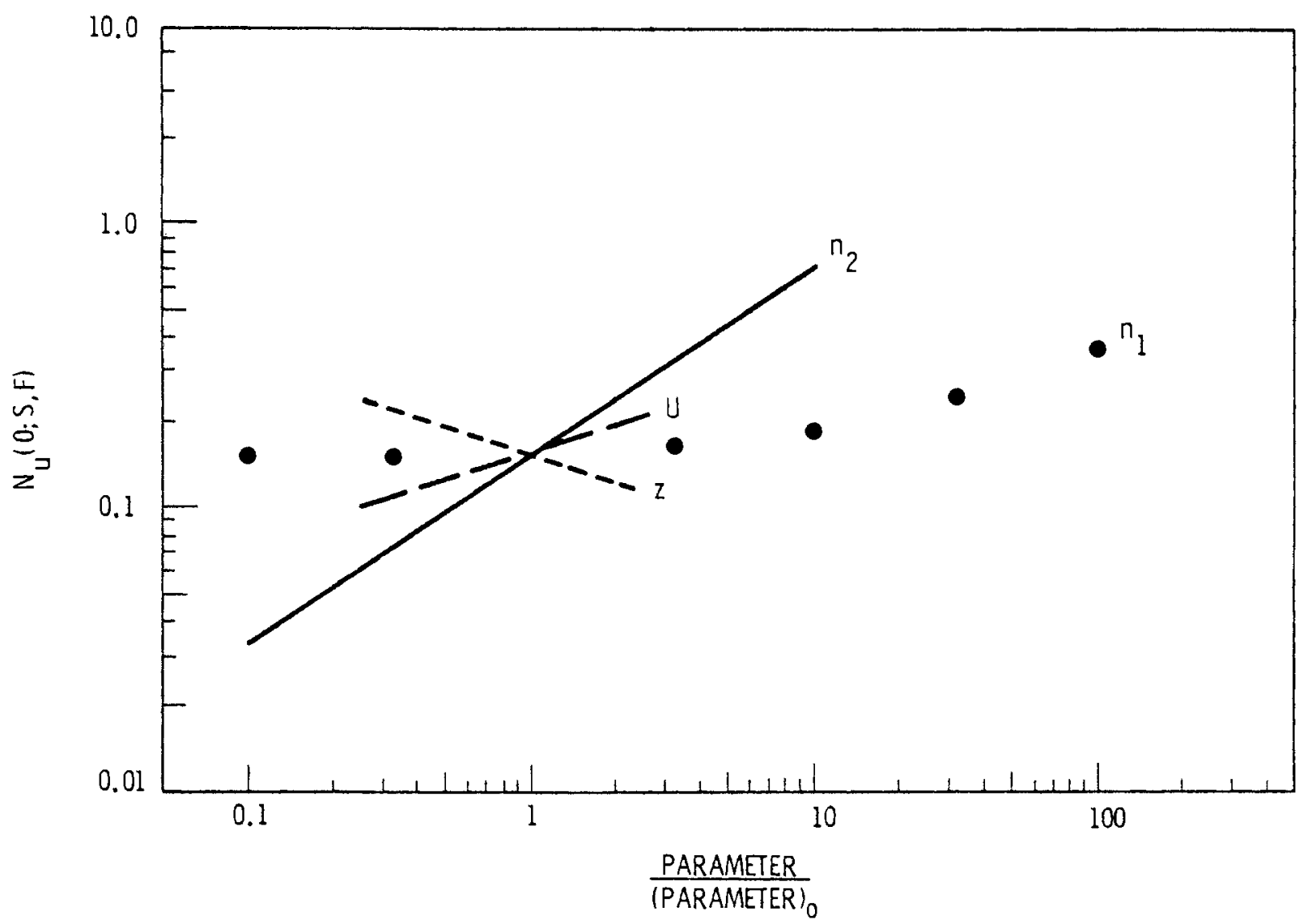

FIGURE 4.1. Dependence of Basic Frequency Factor on Atmospheric and Machine Parameters

show the variation of $N_{u}(0)$ as a function of $n_{1}$. All variations are subject to the original specification of the four variables:

$$
\begin{aligned}
-\quad\left(n_{2}\right)_{0} & =1.0 \mathrm{~Hz} \\
\text { - } \quad(u)_{0} & =12.0 \mathrm{~m} \mathrm{sec}^{-1} \\
\text { - } \quad(z)_{0} & =40.0 \mathrm{~m} \\
\text { - } \quad\left(n_{1}\right)_{0} & =0.001 \mathrm{~Hz} .
\end{aligned}
$$

For this set of data $N_{u}(0)$ is $0.155 \mathrm{sec}^{-1}$. The numbers on the ordinate indicate ratios of 0.1 to 10 in the variation of any of the four variables; i.e., the solid line shows the variation of $\mathrm{N}_{u}(0)$ with $\mathrm{n}_{2} /\left(\mathrm{n}_{2}\right)_{0}$, the dashed line shows variation of $\mathrm{N}_{u}(0)$ with $U /(U)_{0}$, the dotted line shows the variation of 
$N_{0}$ with $z /(z)_{0}$, where $(z)_{0}$ is the original height of $40 \mathrm{~m}$, not roughness length, and the heavy dots show the variation of $N_{u}(0)$ with $n_{1} /\left(n_{1}\right)_{0}$.

Using the specifications listed above as an origin, the variation with each of the first three functions turns out to be surprisingly 1 inear. According to these data, at this origin $N_{u}(0)$ varies approximately with $n_{2}{ }^{0.66}$, with $U^{0.32}$ and with $z^{-0.32}$, (and with $(U / z)^{0.32}$, of course). The variation of $N_{u}(0)$ with $n_{1}$ is unimportant at this origin. At higher values of $n_{1}$, the increase is nonlinear.

Using these relations and the logarithmic wind law,

$$
u\left(z, z_{0}\right)=\left(u_{*} / k\right) \operatorname{Ln}\left(z / z_{0}\right)
$$

where $u_{*}$ is friction velocity, $k$ is von Karman's constant, and $z_{0}$ is surface roughness length, the ratio of $N_{u}(0)$ at the top of a tower $\left(z_{2}\right)$ to that at the bottom $\left(z_{1}\right)$ should be according to the 0.32 power of the following expression:

$$
\frac{[U(z) / z]_{z_{2}}}{[U(z) / z]_{z_{1}}}=\frac{z_{1} \operatorname{Ln}\left(z_{2} / z_{0}\right)}{z_{2} \operatorname{Ln}\left(z_{1} / z_{0}\right)}
$$

If $z_{2}=100 \mathrm{~m}$ and $z_{1}=15 \mathrm{~m}$, this ratio varies from 0.20 to 0.23 as $z_{0}$ varies from $0.05 \mathrm{~m}$ to $0.5 \mathrm{~m}$. Using the 0.32 power we find that $\mathrm{N}_{0}$ at the top of such a tower should be $60 \%$ to $63 \%$ of the value at the lower height.

Turning now to the probability factor $\left.\exp \left[-a x / \sigma_{u}\right)^{b}\right]$, we first remark that there is no theoretical justification for the use of this form (or, for that matter, any other form) for describing the probability distribution of GUST $_{0}$ amplitudes. To derive an empirical justification, then, we refer to the data analysis described in Appendix $B$. The results presented there are very tentative because they are based on analysis of one 55-minute test at one anemometer. Using linear regression on the histogram of GUST amplitudes we find: 


$\begin{array}{lcc} & a & b \\ \text { no filter } & 1.48 & 1.00 \\ 50 / 5 \mathrm{sec} \text { filter } & 0.96 & 1.24\end{array}$

which differ significantly from the values given by the Ramsdell report. A great difference is also made by filtering. Additionally, we know of no theoretical basis for deriving the coefficients for the filtered data from those of the unfiltered data. Such a basis would necessarily involve the spectrum of GUST, events taken as a time series.

Having derived these coefficients, the next step is to fit the GUSTo probability distribution from real data analys is to the form $y=\exp \left[-a\left(x / \sigma_{u}\right)^{b}\right]$ using $a$ and $b$ as given above. The results of this comparison are shown in Figure $4.2 a$ and $b$. Both comparisons show the same systematic deviation of data from model, such that the frequency of occurrence of large amplitude gusts is overestimated. Unless subsequent data analysis supports use of the exponential model, we recommend that the Gaussian model be considered as a first approximation to the probability of GUST $_{0}$ amplitudes, at least for the filtered data. The fit of GUST 0 probability distribution to the Gaussian model is illustrated by the heavy dots in Figure 4.2. This curve follows

$$
P_{A}(x)=\frac{1}{\sqrt{\pi / 2} \sigma_{A}} \int_{x}^{\infty} e^{-\frac{1}{2}\left(\frac{A}{\sigma_{A}}\right)^{2}} d A
$$

where the $P_{A}(x)$ notation indicates the probability factor in Equation (4.1), which also relates back to Equation (2.1). The rms of GUST 0 amplitudes in this equation, $\sigma_{A}$, is used instead of the turbulence rms $\sigma$. For the filtered data the error function fits better than the exponential; for the unfiltered data the exponential is perhaps the better fit. Since we are more concerned with analys is of filtered rather than unfiltered data, our recommendation for the error function (Gaussian) still stands, particulariy 


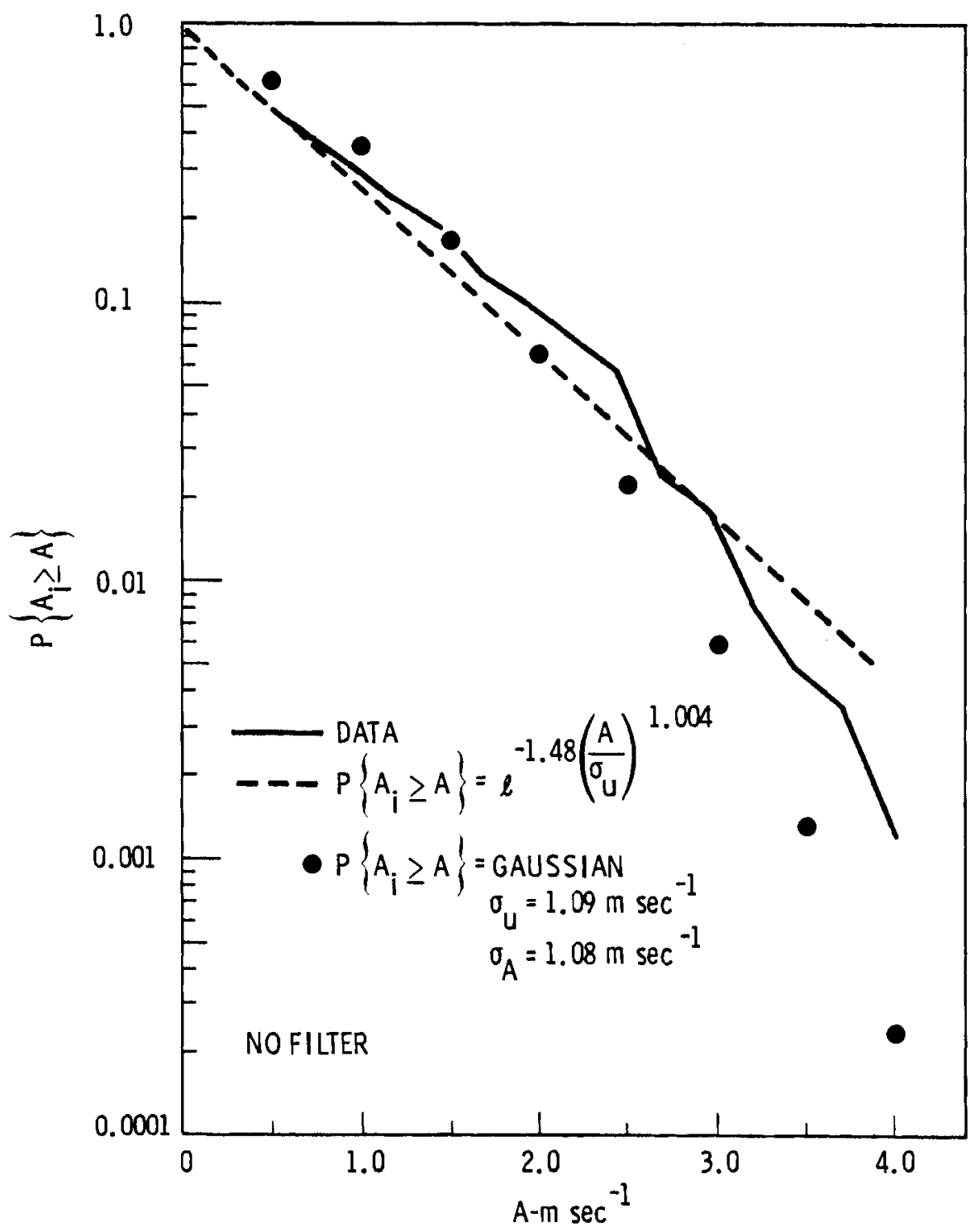

FIGURE 4.2a. Comparison of Observed and Modeled Gust Probability for Ramsdell Gust Analysis Using No Filter 


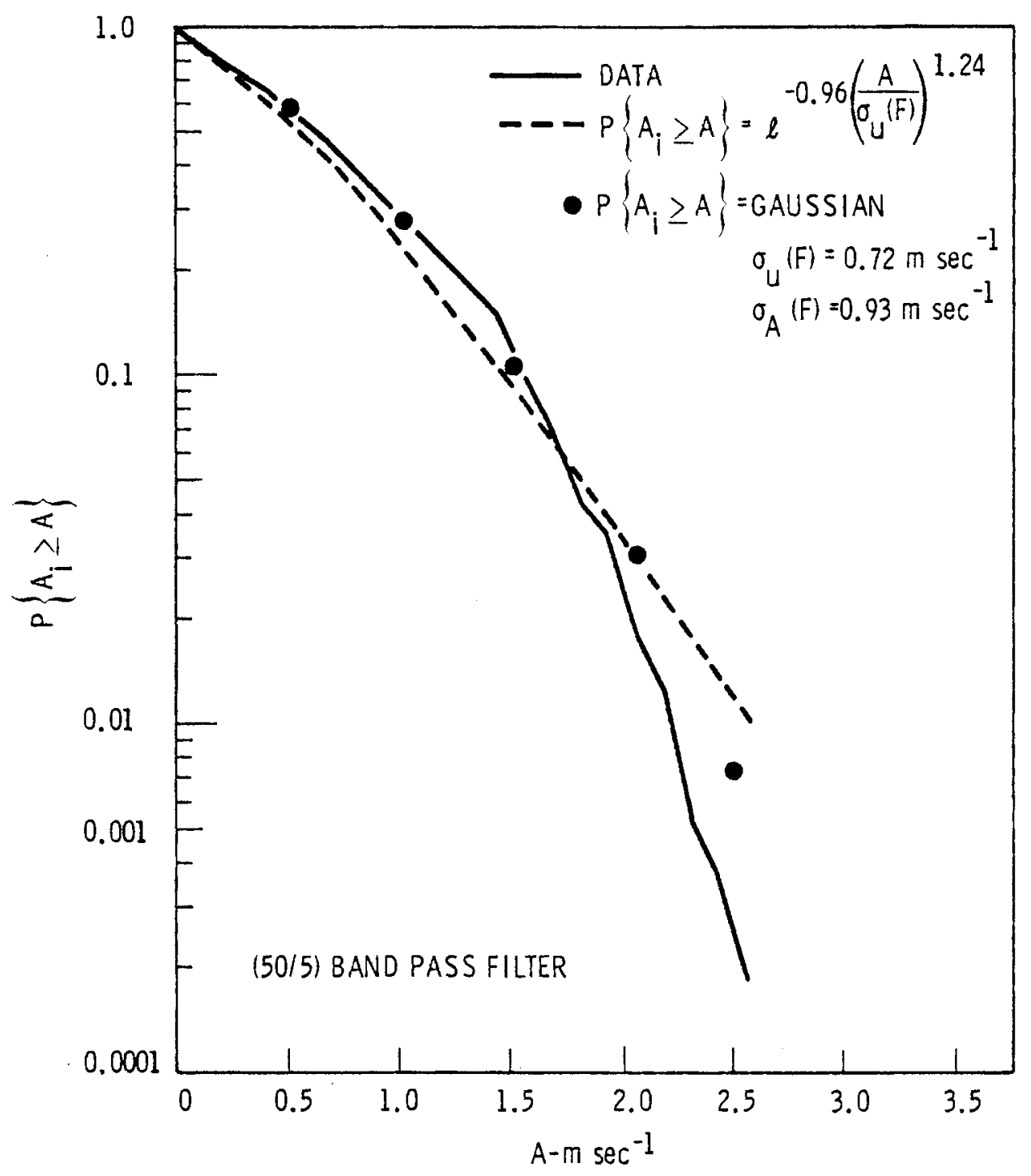

FIGURE 4.2b. Comparison of Observed and Modeled Gust Probability for Ramsde 11 Gust Analys is Using $50 / 5 \mathrm{sec}$ Bandpass Filter 
since the probability factor used in all the other reviewed models is Gaussian. In this way we eliminate one difference among the models that was not well founded.

In order to make a practical model, we need now a relation between $\sigma_{A}$ and $\sigma$, as was spelled out in functional form in Equation (2.1b). This is not simple, since the spectrum of turbulence is involved. The subject is being considered in some additional detail in work at PNL, which extends that shown by Appendix B. In this appendix theoretical and empirical arguments are advanced to tentatively consider the two sigmas as roughly equal for unfiltered data and $\left(\sigma_{A} / \sigma\right)$ equal to 1.3 for bandpass filtered data, when the bandwidth is on the order of one decade.

In summary, we recommend that those who wish to use the Ramsdell GUST $_{0}$ amplitude model for filtered u-component turbulence use instead the GUST 0 model given in Section 6.2 of this report. The derivation of this model from turbulence input is explained in Sections 6.1 and 6.2. In this derivation we assume that the state of the atmosphere is described by the mean wind, i.e.,

$$
S=S(U)
$$

and accordingly the $S$ notation has been replaced by $U$ in the argument lists. The final expression for the GUST, model is Equation (6.13). The recommended turbulence model is that of Equations (6.2) through (6.5). Equations (6.10) and (6.11) are also needed. For rough terrain, the value of $\sigma_{u}$ may be taken from Table 3.1 rather than from Equation (6.2).

Several meteorological assumptions are involved in the GUST 0 model:

- The GUST $_{0}$ amplitudes have a Gaussian distribution. This assumption is fair to good for turbulence data from a bandpass filter one decade wide. The assumption is poor for unfiltered data (see Appendix B).

- Although the surface boundary layer theory involved in the spectrum was derived to fit approximately the lowest $30 \mathrm{~m}$ in the atmosphere, 
the theory can be extended suitably to all heights of interest for WECS machines. This has been partially justified for neutral conditions by statements by Panofsky (1973) indicating that for neutral conditions the theory holds reasonably well to a height of $150 \mathrm{~m}$.

Therefore, the Gaussian part of the proposed model is weak for analysis of unfiltered data. But since the GUST definition has a weak relation to machine responsiveness unless the data are modified at least by highpass filtering, we base our recommendations primarily on results of the analysis of filtered data.

For unfiltered u-component turbulence, we recommend the exponential model in the form that Ramsdell originally conceived it (see Equation (3.4)) with a and b supplied for each application from analysis of onsite data. The values of 1.48 and 1.00 for $a$ and $b$, respectively, appearing in this report are not based on sufficient data analysis.

A designer who has turbulence data from a site of interest and the means of filtering it directly can avoid the spectral estimation of $N_{u}(0)$. Ali he needs to do is filter the data, subtract out the mean value $U$, count the number of positive-slope zero crossings over a time $T_{S}$ and divide by $T_{S}$.

\subsection{THE MOD-2 W.IND TURBINE GUST MODEL}

This model differs formally from the other three because it does not use a basic frequency factor. In the application of this model some designers use WECS blade rotation rate as a basic frequency factor. This, of course, is entirely independent of atmospheric turbulence. In calculating fatigue using this frequency factor, one atmospheric event per blade revolution is assigned on the basis of probability, and one fatigue event is calculated.

A second observation is that the $A$ in Equations (3.15) and (3.16) is multiply defined as gust amplitude and random turbulence values simultaneously. Since turbulence is approximately Gaussian, it is reasonable to regard the $A$ in Equation (3.15) as a turbulence value. In other words, if 
$A$ is defined as a specific value of the random turbulence component $u$, this definition fits the use of $A$ in Equation (3.15). To make this clear, we rewrite Equation (3.15) using the random variable notation described in Section 1.1 .

$$
P_{u}(A ; U, F)=\frac{1}{\sqrt{2 \pi} \sigma_{u}(F)} e^{-\frac{1}{2}\left[\frac{A}{\sigma_{u}(F)}\right]^{2}}
$$

This is very different from the definition of $A$ implied in Equation (3.16), where it is the amplitude of a discrete gust event. Therefore, the $A$ has been doubly defined, and as a result the discrete gust equations are not properly related to the turbulence model of Equations (3.9) through (3.15). The situation can be remedied if the two things that have been defined as one can be related. We use a new notation and rewrite Equation (3.16) as

$$
U(t)=U+A_{c}[1-\cos (2 \pi t / T)] \quad 0 \leq t \leq T
$$

where $t$ is time, $A_{c}$ is the amplitude of a one-minus-cosine gust, and $T$ is gust period. We can interpret $A_{c}$ as being related to the amplitude of a GUST $_{0}$ event (see Figure 2.1). More precisely, if the shape of each gust in Figure 2.1 were modified so that the shape is that of a one-minus-cosine gust, as defined in Equation (4.8), we have

$$
A_{\text {GUST }_{0}}=2 A_{C}, \quad \sigma_{A}=\left(\sigma_{A}\right)_{G U S T_{0}}=2 \sigma_{A_{C}}
$$

A suggested model for GUST 0 amplitudes is given in Section 6.2 of this report. Our recommended model for one-minus-cosine gusts is given in Equation (6.14).

As stated under Equation (3.16), the memorandum specifies that $T$ is the most probable gust time for amplitude $A$. But we have seen no equation that 
we can recommend to describe this relation. From our own ongoing investigation we find that the relation between gust amplitude and typical gust time depends heavily on filtering. Further empirical analys is aimed at relating GUST $_{0}$ time to GUST, amplitude and accounting for the filtering effect is underway at PNL.

The M2 model is again unique among the models reviewed because it recommends the Weibull distribution of mean wind speed. The other three models use the Rayleigh distribution, which is a special case of the Weibull. The Weibull distribution requires specification of a climatological mean wind parameter and a shape parameter. If the shape parameter is frozen, the only degree of freedom left is that of climatological wind speed, which is common to the Rayleigh distribution.

Neither of these mean wind models is unequivocally preferable to the other. However, because of the sensitivity of calculated frequency of occurrence of rare wind fluctuation events to the assumed probability distribution of mean wind, the Weibull distribution is recommended over the Rayleigh if the shape parameter can be confidently specified. This may not be the case if extrapolation is to be made from an airport record to a nearby remote location or even if extrapolation in time is to be made from a short record. The prospective user should see a plot showing how well the Weibull distribution fits a field record in shape before deciding whether or not to use the shape parameter. Neither distribution allows for the case where calms prevail over a significant fraction of the time. In this case, a distribution specified in nonanalytical tabular fashion is recommended.

To sum up, the calculated statistics from the M2 model that we can recommend are the probability of filtered turbulence values, the probability distribution of mean wind speed, and the probability density of gust amplitude if they can be interpreted as half GUST 0 amplitudes and we can write for positive gusts 


$$
p_{A_{c}}(x ; U, F)=\frac{1}{\sqrt{\pi / 2} B \sigma_{u}(F)} e^{-\frac{1}{2}\left(\frac{x}{B \sigma_{u}(F)}\right)^{2}},
$$

where if the band between $n_{1}$ and $n_{2}$ is about a decade, we find

$$
B \approx 0.65,
$$

where

$$
B \sigma_{u}(F)=\sigma_{A_{c}} \quad ;
$$

see Equations (4.9) and (6.11). The difference in the probability densities calculated using this expression and the expression given in the memorandum is shown in Figure 4.3. As can be seen, the difference amounts to orders of magnitude when the gust value is more than twice the turbulence sigma. Since no loss of generality is entailed by setting the turbulence sigma equal to $1 \mathrm{~m} \mathrm{sec}^{-1}$, we have done so in the figure.

\subsection{THE CLIFF-FICHTL AND HUANG-FICHTL MODELS}

Since these two models have marked similarities, they will be treated in the same sub-section. Moreover, the common characteristics will be reviewed in Section 4.3.1, and the differences will be compared in Section 4.3.2.

\subsubsection{Common Characteristics}

The CF and HF models are both velocity-difference models based on the fundamental idea that the change of velocity over a specified period of time is important. In both the turbulence data $u(t)$ is replaced by that of $\Delta u(t, \tau)$ as defined in Equation (3.3), with $\tau$ to be specified. The gust time scale is this $\tau$. The essentials of each model (excluding the wind profile equations in the $\mathrm{HF}$ model) can be derived from assuming a) Rayleigh distribution of mean wind speeds and b) stationary Gaussian turbulence at a given mean wind speed. Both models are intended to predict the frequency of 




FIGURE 4.3. Comparison of Original and Suggested Gust Probability Density

occurrence of extreme velocity-difference magnitudes. Such a frequency of occurrence may range from once per year to once during the projected lifetime of the machine. 
Before reviewing these models separately, four general comments about both models must be made. First, a bias toward underestimating amplitudes is possible if improper values of $\tau$ are used when using the kind of data that applies to both models. This is illustrated in Figure 4.4. If this figure represents $u(t)$ as seen by the machine, and if the change of $u(t)$ as seen by the machine is nonmonotonic throughout the interval $\tau$, then the value assigned to $\Delta u(t, \tau)$ by this definition is less than the maximum difference occurring within this interval. Clearly $\tau$ is too large to represent wind turbine response if the curve does represent the wind to which the turbine responds. However, by using a range of $\tau$ values, some added information about average shapes of gusts may be derivable.

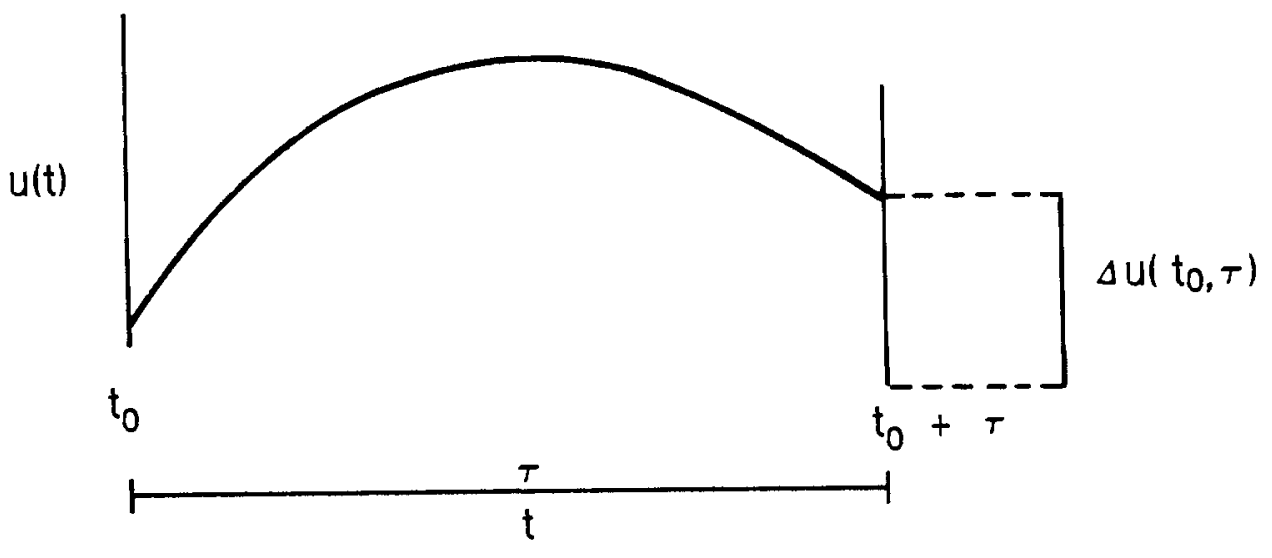

FIGURE 4.4. $\Delta u(t, \tau)$ from Nonmonotonic Change in $u(t)$

The second point concerns extreme magnitudes. The turbulence relations in both models are all derived using the assumption of steadystate turbulence. At extreme gust magnitudes it cannot be assumed that steady-state turbulence prevails, or that a steady state can be defined. Other dynamic processes may be involved (for instance, downdrafts during a thunderstorm) that significantly alter the scales, the spectrum of the turbulence and its probability density. Of course, this objection is less 
critical if mean wind speeds beyond a ceriain cutout speed are not to be considered, i.e., if the final integration over mean wind speed is limited to a finite value that is not extraordinary.

The third point is that at extreme gust magnitudes, the mathematics of these models should be used to solve the inverse problem rather than the direct one. In other words, the extreme gust magnitude that fits a specified recurrence interval should be computed instead of the recurrence interval. In the equation below, which is general enough to fit both models, the solution should be iterated sufficiently to solve for $x$ given $N_{\Delta u(\tau)}\left(x ; F, U_{a}\right)$ rather than the direct way.

$$
\left[N_{\Delta u(\tau)}\left(x ; F, U_{a}\right)=\int_{0}^{U_{C}} N_{\Delta u(\tau)}(0 ; U, F) G\left(\frac{x}{\sigma_{\Delta u}}\right) p\left(U \mid U_{a}\right) d U\right]_{z} .
$$

In this equation the term on the left is the frequency of occurrence of $\Delta u$ values of magnitude $x$. The first term in the integration is a basic frequency factor (which differs fundamentally between the two models), the second factor is a Gaussian turbulence probability, and the third is the probability density of mean wind speed. In this notation we have emphasized that the probability of mean wind speed is a conditional probability depending on the assumed climatological mean wind speed.

The whole point is that at extreme magnitudes the term on the left can vary by orders of magnitude due to uncertainty of 5 or $10 \%$ in $U_{a}$. On the other hand, the extreme gust magnitude associated with a fixed long recurrence interval varies reasonably with variation of input parameters.

A fourth feature common to both models, which we do not recommend, is the use of the Dryden spectrum; see Equations (3.21) and (3.22). This spectrum is false to turbulence in that the high-frequency slope on a log-log plot is -1 rather than the correct $-2 / 3$. This feature of the model may be corrected by using the recommended spectrum of Equations (3.12) through (3.14). The advantage of the Dryden spectrum is that it can be 
integrated analytically when multiplied within the integral by a filter such as that of Equation (3.23). However, in view of the capabilities of hand calculators, this advantage seems unimportant.

A plot showing how well these two spectra coincide depends, of course, on the length scale selected for the Dryden spectrum. If this is fixed so that the two spectra coincide at low frequencies, the high-frequency difference between the two becomes important at values of 0.1 or greater for the dimensionless frequency $f=n z / U$. Since $z / U$ may be on the order of 0.1 , the differences between the two spectra are generally not important at values of dimensional frequency $n$ less than $1 \mathrm{~Hz}$.

We recommend that any designer wishing to use one of these models use the Huang-Fichtl model. In the following comparison of data, discrete events, basic frequency factors and filtering, we show that the HF model is a basically correct version of the exceedance Equation (4.13), while the CF model is not.

\subsubsection{Comparison}

Four features of the HF and CF models are compared: data, discrete events, basic frequency factors and filtering.

\subsubsection{Data}

In the CF model the differencing interval also doubles as a discretization interval. This was shown in Equation (3.17). In the HF model, the discretization interval is arbitrarily small, depending on measuring conditions. Since the HF analysis involves a cut-off frequency that is normally much lower than the Nyquist frequency of the data $(a)$, the unfiltered turbulence time series data is equivalent to a continuous specification of $\Delta u(t, \tau)$ for the subsequent purposes.

\subsubsection{Discrete Events}

The discrete events of these models are illustrated in Figure 4.5. The discrete events of the CF model are discrete values from the continuous (a) Nyquist frequency is the reciprocal of twice the sampling interval. 
DATA: $\Delta u(t, \tau)=u(t+\tau)-u(t)$

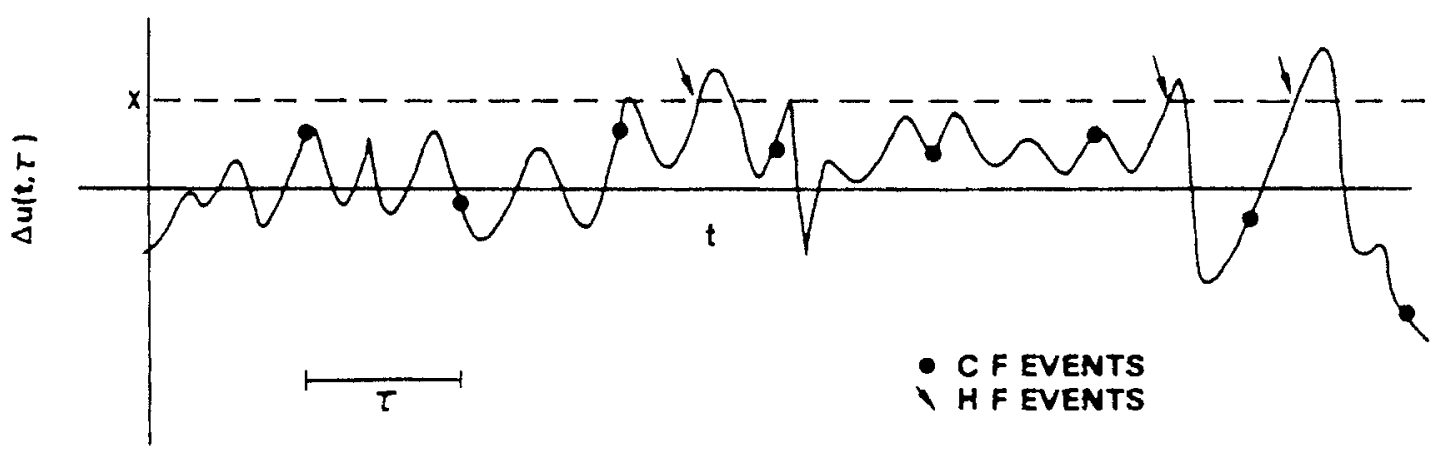

FIGURE 4.5. Discrete Events Defined for Cliff-Fichtl and Huang-Fichtl Models

$\Delta u(t, \tau)$ process separated by the differencing interval $\tau$. These are the heavy dots in the figure. To create discrete events for the HF model, it is necessary to draw a horizontal line at level $x$ through the time function $\Delta u(t, \tau)$ such that the function forms positive-slope level crossings with respect to $x$. These events are indicated by the arrows in the figure. The final discrete events of the CF model also require the line $x$ for definition. They are the large dots at a level exceeding $x$. (None are shown in Figure 4.5.)

4.3.2.3 Basic Frequency Factor

The differences of basic frequency factor between these two models are very significant. Only the HF model uses a basic frequency factor that incorporates atmospheric turbulence behavior. Assuming that the turbulence as seen through the machine filter $F$ is Gaussian, we can write

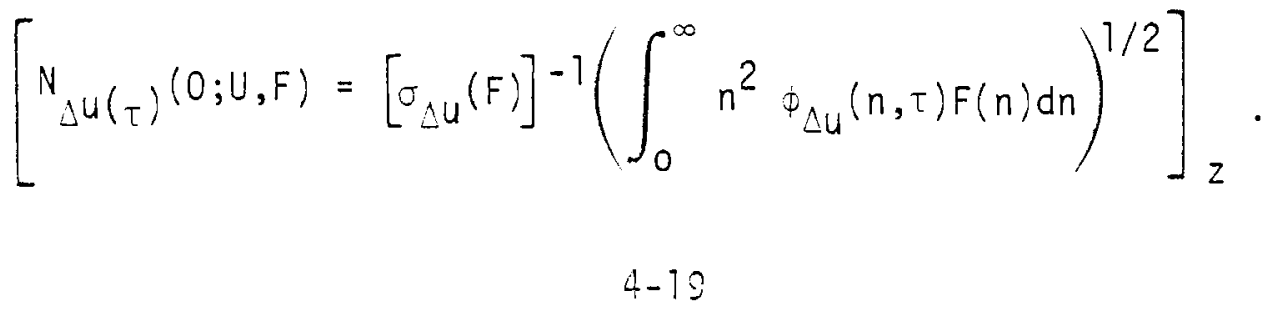


This is a corrected and more general version of the basic frequency factor as given in Equation (3.34), the version in the HF document. Equation (4.14) is more general in that the filtering is unspecified and corrected because the filter is used in the integral of both numerator and denominator. The $U$ dependence on the left comes from the spectrum on the right.

The CF model uses in place of this

$$
N_{0}(\tau)=0.5 / \tau
$$

which is simply the Nyquist frequency that results from the use of the interval $\tau$ as differencing and discretization interval simultaneously. The result is that this model either filters out or aliases (represents the contribution at a lower false frequency) all contribution to the $\Delta u(t, \tau)$ process from frequencies greater than $0.5 / \tau$.

A comparison between these two basic frequency factors is shown in Figure 4.6. This representation is not completely general, since specific input data must be used. But with any reasonable set of input data, the comparison should be the same in that as $\tau$ becomes large, the CF basic frequency factor $0.5 / \tau$ approaches zero while the basic frequency factor, Equation (4.14), oscillates about the zero crossing frequency for the undifferenced series given by Equation (4.2), subject, again, to the assumption that the turbulence, as seen through the filter $F$, is Gaussian.

\subsubsection{Filtering}

Filtering for velocity difference models is 1 imited to lowpass filtering since the differencing is inherently a highpass process. In this respect the CF model better approaches physical reality than does the HF model since the latter uses a simple cutoff frequency while the former uses the gradual reduction of sensitivity expressed by Equation (3.23). However, this formulation lacks definitiveness if for no other reason than the fact that if the filter function is rewritten 


$$
F(n)=\frac{1}{1+\alpha^{2}\left(\frac{n D}{U}\right)^{2}} \text {; }
$$

the generality added by introducing the alpha cannot be collapsed into Equation (3.23) by virtue of any arguments in the CF document.

\subsubsection{Information Available from the HF Model}

Using the HF model, the following data can be calculated where usefulness is evaluated subject to the reservations stated in the beginning of Section 4.3.1:

- frequency with which the $\Delta u(t, \tau)$ process exceeds any designated value (frequency of level crossings)

- fraction of time over which $\Delta u$ exceeds any given value

- average duration of an exceedance interval for any level $x$.

Only the first of these is given explicitly in the document. This is calculated using Equations (3.33) through (3.36); however, we suggest that in Equation (3.34) the cutoff frequency used in the numerator also be used in the denominator. A specification of mean wind probability, which in the HF model is the Rayleigh distribution, Equation (3.30), is also needed. One may deduce from the same set of equations that the fraction of time $R_{\Delta u(\tau)}(x ; F)$ over which the $\Delta u(t, \tau)$ exceeds some positive value $x$ is

$$
R_{\Delta u(\tau)}(x ; F)=\int_{0}^{U_{C}} \frac{1}{\sqrt{2 \pi} \sigma_{\Delta u}(F)}\left[\int_{x}^{\infty} e^{-\frac{1}{2}\left[\frac{\Delta u}{\sigma_{\Delta u}(F)}\right]^{2}} d \Delta u\right] p(U) d U .
$$

Then the average duration $D_{\Delta u(\tau)}(x ; F)$ of each exceedance interval is

$$
\left[D=\frac{R}{N}\right]_{\Delta u(\tau)}(x ; F)
$$




\subsection{RECOMMENDATIONS FOR FUTURE MODELING}

This section is addressed primarily to those who are interested in further wind fluctuation model development. The reader who is interested in using rather than developing models may wish to skip to section 6 at this time.

Section 6 begins by substantiating the statement that no one gust model can be recommended as being universal at this time. Therefore, recommendations for future modeling are addressed to more than one kind of windfluctuation model.

\subsection{FOR FUTURE. TURBULENCE MODELING}

We have designated the portion of the M2 model that appears in Equations (3.9) through (3.15) of this report as a turbulence model. The information from the turbulence model can be extended by adding calculations of frequency of level crossings. The theoretical basis of this work is discussed below in relation to Equation (4.2). The equations for this calculation also result if the equations of the HF model are changed so that the data are those of $u(t)$ rather than those of $\Delta u(t, \tau)$.

Using Equation (4.2) to calculate the frequency of positive-slope zero crossings and letting the mean wind speed $U$ represent the state of the a tmosphere $S$, we write

$$
\begin{aligned}
N_{u}(x ; U, F) & =N_{U}(O ; U, F) e^{-\frac{1}{2}\left[\frac{u}{\sigma_{u}(F)}\right]^{2},} \\
N_{u}(x ; F) & =\int_{0}^{U}{ }^{C} N_{U}(x ; U, F) p(U) d U
\end{aligned}
$$

where the specification of the probability density of mean wind $p(u)$, be it a Rayleigh or Weibull distribution, is really outside the scope of the turbulence model. 
This type of model was al ready suggested by Frost et al. (1978; Section 4.3). The title of this section ("Spectral Model") fits this model very well since it is derived from spectral consideration and has nothing to do with an independent discrete gust definition.

The filtering concept involved in this model is governed by the assumption that only fluctuations that engulf the entire rotor should be considered. Vertical and lateral coherence are used in defining the filter function in Equation (4.2).

\subsection{FOR FUTURE DISCRETE GUST MODELING, INCLUDING VELOCITY-DIFFERENCE MODEL}

By discrete gust modeling, we restrict ourselves to the meaning of a mathematically stated approximation of the relevant physical processes. From our point of view, a primary requirement for discrete gust model ing is that a definition of discrete gusts, or discrete events, be adopted that clarifies the items related to gusts that atmospheric science has not previously defined and that this definition thereafter be used in a consistent manner, verbally and conceptually. Otherwise there can be no commonly accepted basis for scientific evaluation.

The definitions applied to wind fluctuation description by atmospheric scientists are usually those of turbulence theory. These presume a stationary process that is horizontally homogeneous. Since these definitions are independent of any definition of discrete events within turbulence, or turbulence time series, the addition of a discrete event definition appears to be the natural beginning for the building of models of discrete events, or discrete gusts. Such a definition was proposed in Section 2.1 of this report.

Another step, if discrete event modeling is to be a science, is to find out what information becomes available by virtue of the new discrete event definitions that is not already available as turbulence information (for example, information about a stationary, homogeneous process) or easily derived from turbulence information. The discrete events definition of Section 2.1 involves a velocity scale and a time (gust period) scale. Are these independent of velocity and time scales of turbulence? How are these 
related to velocity and time scales of turbulence? From work that is ongoing at PNL we can offer some initial observations. These are given in Appendix $B$.

A third point is that statistics of joint probability of amplitudes and time intervals of discrete event definitions should be offered to the designers for their evaluation. This work is now ongoing at PNL using the GUSTO definition. This work will emphasize the conditional probability of gust time given gust amplitude and the conditional probability of gust amplitude given gust time. The ranges of amplitude and time that contribute to the high overall correlation

$$
0.67 \leq \rho_{A T} \leq 0.76
$$

will be discussed.

The fourth observation applies to the fundamentally different type of velocity difference model that uses for its data a differenced velocity series with differencing at an arbitrary interval as defined by Equation (3.3). In Section 4.3 we raised the objection that this model underestimates amplitudes because the velocity change within the interval may not be monotonic; see Figure 4.3 and discussion. We recommend further assessment of the seriousness of this feature.

\subsection{REGARDING STATIONARY OR MOVING FRAMES OF REFERENCE}

All the reviewed models are Eulerian and are based on the idea that application of filters to a time series can produce an analysis that simulates the responses of a machine to "engulfing gusts." Arguments can also be advanced to the effect that wind fluctuations as seen from points rotating like points on a WECS blade are in some cases the relevant data. This type of analysis is being pursued at PNL both spectrally and using discrete event definitions. 


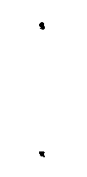




\subsection{CONCLUSIONS}

We conclude that no one gust model can be recommended at this time. We are constrained by the existing lack of agreement among designers and atmospheric scientists as to which descriptors are needed in a gust model. However, from the materials introduced in this report we can assemble three different models, each of which handles a distinctly different descriptor.

A common element for a 11 models is extrapolation of mean wind from reference height to hub height; for this purpose a wind profile equation is needed. Over complex terrain this may require empirical determination. over homogeneous terrain, the logarithmic wind law is applicable in neutral stability up to about $150 \mathrm{~m}$. This is given by

$$
\frac{U(z)}{U\left(z_{r}\right)}=\frac{\operatorname{Ln}\left(z / z_{0}\right)}{\operatorname{Ln}\left(z_{r} / z_{0}\right)}
$$

where $z_{r}$ is reference height and $z$ is hub height. Modifications of this law for stable or unstable atmospheric stratification are available in atmospheric science literature; but, they are of limited applicability for field studies since they contain terms that require turbulence data to evaluate. For these conditions, power laws such as those discussed by Frost et al. (1978) are probably the most useful and congenial representations of wind profiles for designers.

Another common element of all models is the probability density of mean wind speed at a reference height. Because of the sensitivity of the calculated frequency of occurrence of unusual gust magnitudes to the assumed mean wind speed distribution, we recommend that no shortcuts be used in this part of the model. We recommend three probability densities of mean wind in the following order:

1) a tabular density of frequency with which the mean wind speed occurs in mean wind speed classes, if this is available from high-quality data taken from a site 
2) the Weibull probability distribution, Equation (3.9), if the data base is such that two empirical constants may be determined with confidence

3) the Rayleigh density, Equation (3.30), if neither of the above applies.

In the specification of three models, we consider only u-component turbulence and assume that a model for $u$-component turbuience does not differ significantly from a model of horizontal wind speed; see Appendix $B$.

\subsection{TURBULENCE MODEL}

Let the relation of turbulence to mean wind be

$$
\sigma_{u}=\frac{u(z)}{\operatorname{Ln}\left(\frac{z}{z_{0}}\right)}
$$

In complex terrain Equation (3.5) and Table 3.1 should be used instead. The recommended turbulence spectrum is

$$
\begin{aligned}
n \phi_{u}(n) & =\frac{0.164 \sigma_{u}^{2}\left(f / f_{o u}\right)}{1+0.164\left(f / f_{\text {ou }}\right)^{5 / 3}} \\
f & =n z / U \\
f_{\text {ou }} & =0.0144
\end{aligned}
$$

Let the filtering properties of a machine $F(n)$ be represented by

$$
F(n) \begin{cases}=1 & n_{1} \leq n \leq n_{2} \\ =0 & \text { otherwise. }\end{cases}
$$


Then the rms of filtered turbulence $\sigma_{u}(F)$ is given by

$$
\sigma_{u}(F)=\left[\int_{n_{1}}^{n_{2}} n \phi_{u}(n) F(n) d \operatorname{Ln}(n)\right]^{1 / 2} .
$$

In the four equations, we wish to distinguish between calculations that apply at a given mean wind speed $U$ and those that involve integration over $U$ using the probability density $p(U)$. Therefore, we add $U$ to the argument notation, recognizing that $U$ is also rightfully an argument of Equations (6.2), (6.4), and (6.5). If we assume that the turbulence is Gaussian, the probability of encountering positive value of u-component filtered turbulence that exceeds the positive value $x$ is

$$
P_{u}(x ; U, F)=\frac{1}{\sqrt{\pi / 2} \sigma_{u}(F)} \int_{x}^{\infty} e^{-\frac{1}{2}\left(\frac{u}{\sigma_{u}}\right)^{2}} d u
$$

In principle the probability integrated over all values of mean wind speed is

$$
P_{u}(x ; F)=\int_{U_{1}}^{U_{2}} P_{u}(x ; U, F) p(U) d U
$$

where $U_{1}$ and $U_{2}$ may be cut-in and cutout speeds of a WECS machine, or may be zero and infinity.

The frequency of positive-slope level crossings at level $x$ by the same turbulence function is given by 


$$
\left.\begin{array}{l}
N_{u}(x ; U, F)=N_{u}(0 ; U, F) e^{-\frac{1}{2}\left(\frac{x}{\sigma_{u}(F)}\right)^{2}} \\
N_{u}(0 ; U, F)=\left[\sigma_{u}(F)\right]^{-1}\left[\int_{n_{1}}^{n_{2}} n^{3} \phi_{u}(n) F(n) d[\operatorname{Ln}(n)]\right]^{1 / 2} \cdot
\end{array}\right\}
$$

Integrating over values of $U$, we obtain

$$
N_{u}(x ; F)=\int_{U_{1}}^{U_{2}} N_{u}(x ; U, F) p(U) d U .
$$

There are three final comments to make about this model. First, it is assembled from materials found in the Spera-Richards memorandum (see Appendix A) and from materials in Section 4.3 of Frost et al. (1978). A more refined version of this model may be available shortly from these authors. Second, the inclusion of Equations (6.7) and (6.9) is for form only. The use of Equations (6.7) and (6.9) to predict for very unusual magnitudes is questionable since these most likely do not derive physically from the stationary processes that a turbulence model represents. Third, we prefer to call this a turbulence model rather than a discrete gust model because the only discrete events involved are level crossings, and al though they have a velocity measure, they have no time measure. The turbulence model is a spectral model that incorporates Gaussian probability for the turbulence.

\subsection{DISCRETE GUST MODEL FOR FILTERED DATA}

GUST $_{0}$ events are delimited by adjacent crossings of the mean vaiue of a time series as shown in Figure 6.1. Each GUST 0 event is tagged with amplitude $A$ and time $T$. 


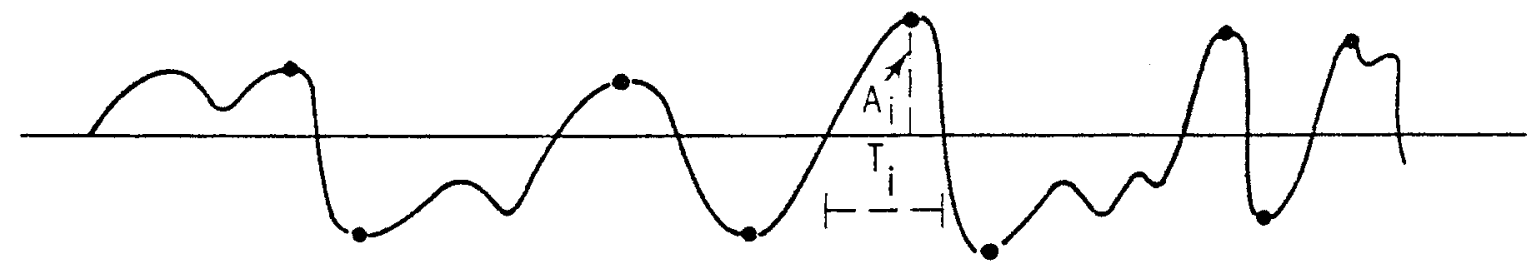

FIGURE 6.1. GUSTO Definition

The GUST 0 model here is completely specified only in terms of positive amplitude. The joint relations between amplitude and time are being investigated in present work at PNL.

The discrete GUST 0 model uses the mean wind and turbulence model of Equations (6.1) through (6.4). We define the rms of gust amplitude $\sigma_{A}$ and of gust time $\sigma_{T}$, and at the present stage of development of the model we must assume that the bandwidth indicated by Equation (6.4) is on the order of a decade, i.e.,

$$
n_{2} / n_{1} \sim(10)
$$

If this holds, we find that both amplitudes and times have a distribution that approaches Gaussian, and we can write, tentatively,

$$
\begin{gathered}
\sigma_{A}(F) \approx 1.3 \sigma(F) \\
\sigma_{T}(F) \approx 0.65 / N_{u}(0 ; F)
\end{gathered}
$$

If the bandwidth approaches zero, the coefficient in Equation (6.11) necessariiy approaches 1.4 , asymptotica $17 y$. If the bandwidth approaches infinity, the coefficient in Equation (6.11) drops toward unity, or even less if the turbulence is notably unstationary. Both gust amplitude and gust time lose their Gaussian probability under either of these conditions. 
Assuming that we can use Equation (6.11), we can write the exceedance frequency of GUST $_{0}$ events as

$$
N_{A}(x ; U, F)=N_{u}(0 ; U, F) \frac{1}{\sqrt{\pi / 2} \sigma_{A}(F)} \int_{x}^{\infty} e^{-\frac{1}{2}\left(\frac{A}{\sigma_{A}(F)}\right)^{2}} d A
$$

where $N_{u}(0 ; U, F)$ taken from Equation (6.8), and where $x$ is non-negative.

Also, with the reservations explained above, we can integrate over mean wind speed. Integrating the exceedance frequency over mean wind speeds involves the probability density of mean wind as in Equation (6.9) and, as explained above, cannot be recommended for unusual gust magnitudes occurring outside the stationary processes to which the turbulence equations apply.

This model is assembled from the turbulence model in the SperaRichards memorandum (Appendix $A$ ) and from the zero-level crossing equation of Rice (1945) and from the analysis in Appendix B. For analysis of filtered data, it is recommended as a replacement of the Ramsdell model; see Section 4.1 .

We can also write a version of Equation (6.13) for positive one-minuscosine gusts of amplitude $A_{C}$ is the relation between GUST 0 amplitudes and rms if GUST $_{0}$ and one-minus-cosine gust description can be equated as in Equation (4.9). If so, we can write

$$
\left.\begin{array}{c}
N_{A_{C}}(x ; U, F)=N_{u}(0 ; U, F) \frac{1}{\sqrt{\pi / 2} \sigma_{A_{C}}(F)} \int_{x}^{\infty} e^{-\frac{1}{2}\left(\frac{A_{C}}{\sigma_{A_{C}}(F)}\right)^{2}} \\
\sigma_{A_{C}}(F) \approx 0.65 \sigma_{u}(F)
\end{array}\right\}
$$

where $N_{u}(0 ; S, F)$ is taken from Equation (6.8), and where $x$ is non-negative. 


\subsection{VELOCITY-DIFFERENCE (GUST-RISE) MODEL}

This type of model uses differenced velocity data where the differencing interval is specified by the analyst. The differenced data is

$$
\Delta u(t, \tau)=u(t+\tau)-u(t) \quad 0 \leq t \leq T_{s}
$$

where $T_{s}$ is a period over which a mean wind $U$ may be defined. The spectrum of $\Delta u$ is

$$
n \phi_{\Delta u}(n, \tau)=2 n \phi_{u}(n)(1-\cos 2 \pi n \tau),
$$

and $\phi_{u}(n)$ is given by Equation (6.3). The rms of the filtered $\Delta u(t, \tau)$ process is

$$
\sigma_{\Delta u(\tau)}(F)=\left[\int_{0}^{\infty} \phi_{\Delta u}(n, \tau) F(n) d n\right]^{1 / 2}
$$

where $F(n)$ is given by Equation (6.4). Then the frequency of positive-slope crossings of level $x$ by the $\Delta u(t, \tau)$ process is

$$
\begin{gathered}
N_{\Delta u}(x ; U, F)=N_{\Delta u}(0 ; U, F) e^{-\frac{1}{2}\left(\frac{x}{\sigma_{\Delta u}(F)}\right)^{2}} \\
N_{\Delta u}(0 ; U, F)=\left[\sigma_{\Delta u}(F)\right]^{-1}\left[\int_{0}^{\infty} n^{3} \phi_{\Delta u}(n, \tau) F(n) d[\operatorname{Ln}(n)]\right]^{1 / 2} .
\end{gathered}
$$

With the usual reservations, we mention that Equation (6.19) can be integrated over mean wind speeds if the probability density $p(U)$ is supplied. 
This model is virtually the same as the Huang-Fichtl model. The only significant difference is that we recommend that the turbulence treatment from the M2 model supplant that in the Huang-Fichtl model.

\subsection{COMMENTARY ON THE ABOVE THREE MODELS}

A stationary Gaussian process must be assumed for application of any of these models. This assumption is violated less seriously by the turbulence model than by the other two. (The validity of application of the models is a separate question not addressed here.) Under stationary conditions the kurtosis of turbulence is slightly greater than the Gaussian value of 3 ; the positive departure of the turbulence kurtosis from 3 is magnified in the departure of the kurtosis of the differenced data. Therefore, under stationary conditions the turbulence model satisfies the Gaussian assumption better than does the velocity difference model. When the turbulence kurtosis is low, due to nonstationarity in the form of large low-frequency waves, the GUST, kurtosis is large and departs more from the Gaussian turbulence than does the turbulence kurtosis. Therefore, under nonstationary conditions involving large low frequency content, the turbulence model aspects of the real atmosphere satisfy the Gaussian assumption better than do the GUST $_{0}$ model aspects of the real atmosphere. Another way to state this is to recognize that the theoretical usefulness of any of these models requires that the filtered turbulence or gust data be of approximately Gaussian probability. Because of the rate at which kurtosis changes with filtering, this restriction is least serious for the turbulence model and most serious for the GUST, model. However, on the basis of the empirical GUST, analysis we have conducted so far, we believe that this requirement (inherent in the theoretical model but not required in the empirical model) can be met, particularly if a bandpass filter of a decade width is not much different from the filter appropriate for the machine. (If not, then present theory simply fails and observation must stand alone.)

The GUST $_{0}$ model also has the property that the turbulence data are unequally weighted in the GUST, statistical analysis. This is easily seen if we imagine a time series with one long GUST 0 event followed by several 
short ones occupying a span of time equal to that of the long event. The long event gets one "vote" in the GUST, analysis, while the equal segment of time series occupied by several small GUST events gets several "votes." of course, this inequality of representation is minimized when a highpass or bandpass filter is applied. This statement is made because application of the filter is simultaneously desirable in two ways. First, it renders the data more as the turbulence is seen by the machine, and second, the statistics are usually more Gaussian and thus more amenable to analysis. A further complication (but perhaps an advantage) of the GUST model is that the relations between gust and turbulence scales, the quantities $\sigma_{A} / \sigma_{U}$ and $\sigma_{T} N(0)$, are also functions of the filtering. Any function of filtering is also a function of the turbulence spectrum, and at present we have no analytical form for the needed function. The major advantage of the GUST model is that description of the joint probability of gust amplitude and gust time is available from this descriptor, alone, of those we have presented.

The principal disadvantage of the velocity-difference model is that the severity of the effect of the gust environment may be underestimated because the $\tau$ may be improperly chosen and the change of $u(t)$ may not be monotonic during the interval $\tau$ or a single $\tau$ may not be adequately representative of the features of gusts that the designer wishes to extract; see Figure 4.3 . Some quantification of the seriousness of this defect is needed.

One final reservation common to all of these models is that they do not apply to nonstationary conditions, i.e., mountain waves and extreme conditions such as frontal passages, thunderstorms and worse storms during which a stationary condition cannot be defined but which may be the sources of extreme amplitude gusts that wind turbines would experience.

\subsection{COMMENTARY ON DISCRETE GUST MODELING AS AN IDEA}

The theoretical idea of this report has been to show what develops when a discrete events definition, including simultaneous application of velocity and time tags, is superimposed on turbulence definitions. We conclude that when the discrete events are GUST, events, we have shown the following: 
1. The discrete event model is a distinct model with spectral input.

2. The discrete event analysis of the wind is more difficult and in ways is more strongly influenced by filtering than is turbulence analysis.

3. The joint probability analysis of amplitude and time will require empirical description, at least initially, and therefore must be based both on a computer program and a large data base for which there is sufficiently documented quality assurance.

4. Discrete gust analysis can provide useful information that is lost in a spectral analysis. 


\subsection{REFERENCES}

Batchelor, G. K. 1953. The Theory of Homogeneous Turbulence. Cambridge University Press.

Cliff, W. C., and G. H. Ficht1. 1978. Wind Velocity-Change (Gust Rise) Criteria for Wind Turbine Design. PNL-2526, Pacific Northwest Laboratory, Richland, Washington.

Counihan, J. 1975. "Adiabatic Atmospheric Boundary Layers: A Review and Analysis of Data from the Period 1880-1972." Atmospheric Environment, $9: 877-905$.

Frost, W., B. H. Long and R. E. Turner. 1978. Engineering Handbook on the Atmospheric Environmental Guidel ines for Use in Wind Turbine Generator Development. NASA TechnicaT Paper 1359, NationaT Aeronautics and Space Administration, Washington, D.C.

Huang, C. H., and G. H. Ficht1. 1979. Gust-Rise Exceedance Statistics for Wind Turbine Design. PNL-2530, Pacific Northwest Laboratory, Richland, Washington.

Kaimal, J. C. 1973. "Turbulence Spectra, Length Scales, and Structure Parameters in the Stable Surface Layer." Boundary Layer Meteorology, $4: 289-309$.

Kerrigan, T. C. 1977. Spectral Estimates of a Wind Fluctuation Statistic Pertaining to Wind Energy Generators. PNL-2511, Pacific Northwest Laboratory, Richland, Washington.

Panofsky, H. A. 1973. "The Boundary Layer Above $30 \mathrm{~m}$. " Boundary Layer Meteorology, 4:251-264.

Powel1, D. C. 1979. Review of Gust Model Concepts. PNL-SA-7966, Pacific Northwest Laboratory, Richland, Washington.

Ramsdel1, J. V. 1975. Wind and Turbulence Information for Vertical and Short Take-off Landing (V/STOL) Operations - Results of Meteorological Survey. Report No. FAA-RO-75-94, Dept. of Transportation, Federal Aviation Administration Systems Research and Development Service, Washington, D.C.

Ramsde11, J. V. 1978. Estimates of the Number of Large Amplitude Gusts. PNL-2508, Pacific Northwest Laboratory, Richland, Washington.

Rice, S. 0. 1945. "Mathematical Analysis of Random Noise." Bell System Technical Journal, 24(1):46-156. 


\subsection{GLOSSARY}

A

$\bar{A}$

$A_{C}$

CF

D

E

$F(n)$

$F(n, \tau)$

f

fo

fou

GUST $_{O}$

GUST, $_{1}$

HF velocity amplitude of a discrete event (exception, Sections 3.2 and 4.2 in relation to $M 2$ model usage) (length time ${ }^{-1}$ ) average value of $A$ (length time ${ }^{-1}$ ) amplitude of a one-minus-cosine gust (length time ${ }^{-1}$ )

gust model by W. C. Cliff and G. H. Fichtl

diameter of rotation circle of a WECS blade (length)

generalized discrete turbulence events; also the expectation operator

power spectral filter in frequency space, simulating machine responsiveness (dimensionless)

same as $F(n)$ but incorporating the filtering characteristics of differencing a time series over interval $\tau$

dimensionless frequency $n z / \bar{U}$

characteristic value of dimensionless frequency $f$ used to shift the frequency of a power spectral model (dimensionless)

$f_{0}$ in $u$-component turbulence spectrum (dimensionless)

discrete turbulence events defined between mean value crossings in a turbulence time series; see Figure 2.1 (length time ${ }^{-1}$, time)

discrete turbulence events defined between zeroes of the first derivative in a turbulence time series; see Figure 2.1 (length time ${ }^{-1}$, time)

gust model by $C$. H. Kuang and G. H. Fichtl. 
$\mathrm{Hz}$

$K_{u}, K_{A}, K_{T} \quad$ kurtosis of $u, A$, or $T$ (dimensionless)

$L_{u}$

M2

N

$N_{A}(x)$

$N_{A T}(x, y)$

$N_{0}$

$N_{0}(\tau)$

$N_{u}(0)$

$N_{\Delta u}(0)$

n

$n_{1}, n_{2}$

$n \phi(n)$

P

$P_{A}(x)$

MOD-2 wind turbine gust mode1

dimensionless frequency $\mathrm{nL}_{\mathrm{u}} / \mathrm{U}$ exceed the value $x\left(t_{i m e}{ }^{-1}\right)$ (gust-rise) models (time ${ }^{-1}$ ) a particular form of $N_{0}(\tau)\left(\right.$ time $\left.^{-1}\right)$

cyclical frequency (cycle time ${ }^{-1}$ ) machine sensitivity (cycle time ${ }^{-1}$ ) expression
$\operatorname{Hertz}\left(\sec ^{-1}\right)$

integral length scale of turbulence (length)

frequency of occurrence of discrete gust amplitudes $A$ that

frequency of occurrence of discrete gusts with amplitude $A$ exceeding $x$ and time period $T$ exceeding $y\left(t_{i m e}{ }^{-1}\right)$

generalized basic frequency factor $\left(\right.$ time $^{-1}$ )

generalized basic frequency factor for velocity-difference

frequency of positive-slope zero crossings by u-component turbulence -- a particular form of $N_{0}$ (time ${ }^{-1}$ )

frequency of positive-slope zero crossings by $\Delta u(t, \tau)$--

lower and upper limit frequencies of a frequency band of

logarithmic power spectrum (1ength ${ }^{2}$ time $^{-2}$ )

dimensionless probability used in frequency of occurrence

probability that a discrete gust amplitude $A$ will exceed $x$ (dimensionless) 


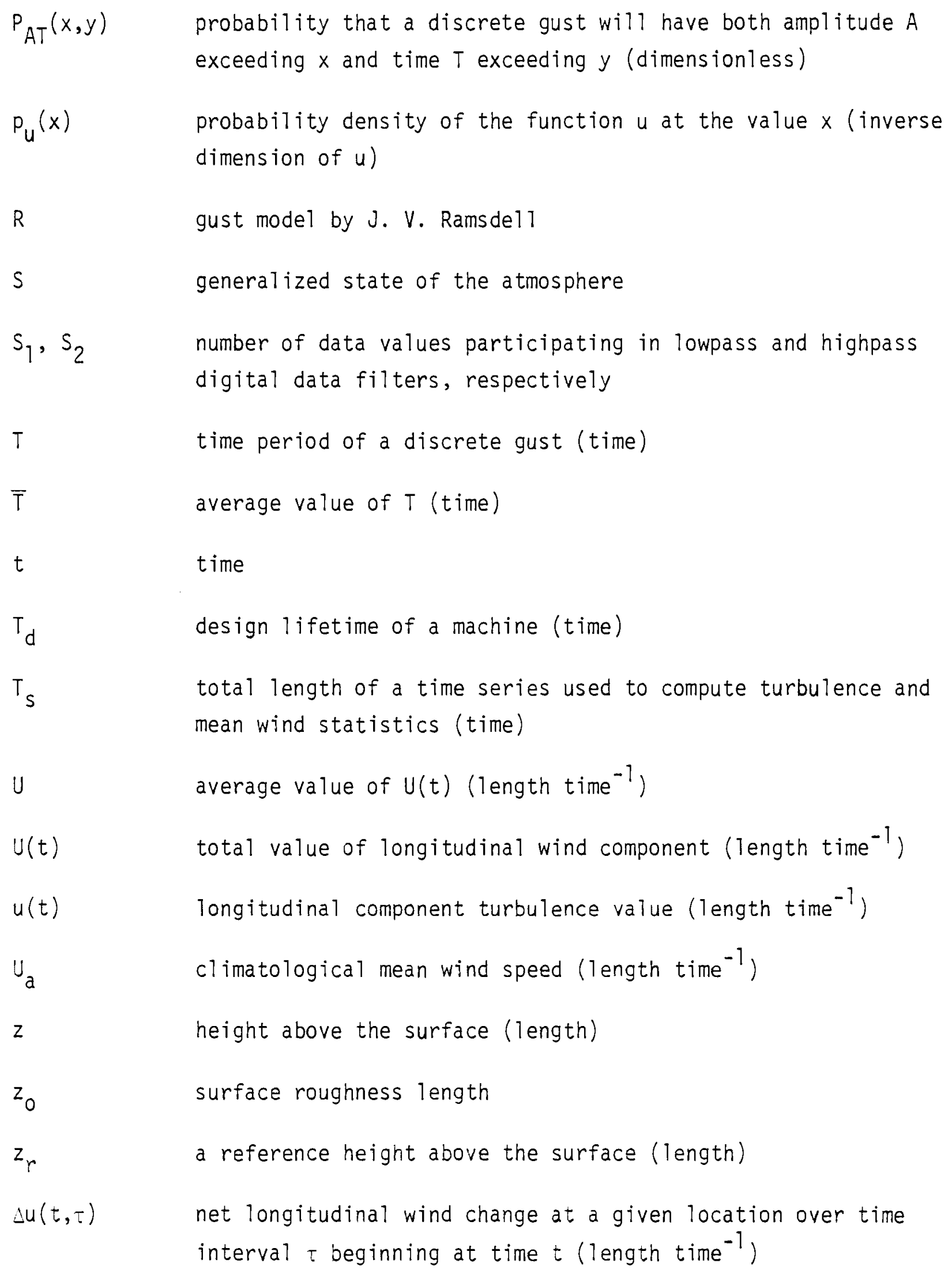


$\rho_{A T}$

$\sigma$

$\sigma_{A}$

$\sigma_{\top}$

$\tau$

$\phi(n)$

$\phi(n, \tau)$ normalized cross correlation of gust amplitude and gust time increment of either GUST $_{0}$ or GUST 1 (dimensionless)

turbulence rms (length time $^{-1}$ )

rms of gust amplitudes A (length time ${ }^{-1}$ )

rms of gust time increments (time)

differencing time - time interval used in subtracting a time series from itself to form such data as

$$
\Delta u(t, \tau)=u(t+\tau)-u(t)
$$

(time)

power spectral density of turbulence (1ength ${ }^{2}$ time $^{-1}$ )

power spectral density of a differenced time series such as $\Delta u(t, \tau)$ (length ${ }^{2}$ time $^{-1}$ ) 


\section{APPENDIX A}

WIND GUST ANALYSIS FOR WIND TURBINE DESIGN 
APPENDIX A

\section{WIND GUST ANALYSIS FOR WIND TURBINE DESIGN}

David A. Spera and Timothy R. Richards (a)

\section{INTRODUCTION}

Wind gusts are one of several important sources of cyclic and transient loading in wind turbines. To be economical, major wind turbine components must be able to accept these unsteady loads without fatigue failure during rather long service lives, on the order of 10 to 30 years. Therefore, it is important that we develop and improve methods for predicting the size and duration of wind gusts, as well as the response of wind turbine systems to these gusts.

The purpose of this paper is to define wind gusts in terms which are useful to the designers of wind turbines and which, at the same time, respect the stochastic nature of the wind. At the present time, deterministic modeling of the response of horizontal-axis wind turbines to wind loads is in an advanced state of development (Ref. 1). However, comparison of deterministic predictions of dynamic loads with actual loads measured in the DOE/NASA Mod-0 wind turbine (Ref. 2) clearly shows that stochastic effects cannot be neglected.

To include stochastic wind effects, the designer must have information of three types: (1) generalized equations defining both the steady and turbulent characteristics of the wind, (2) site-specific wind data, and (3) the response data on the specific wind turbine under analysis. The scope of this paper is 1 imited to the first of these three items. The remaining items are part of the iterative analysis/design process.

The primary source of the gust modeling data presented in this paper is a handbook compiled by Frost and Long (Ref. 3 ).

(a) NASA-Lewis Research Center 
In most cases, the terminology of Reference 3 has been maintained in the equations presented in this paper.

APPROACH

First, the annual distribution of the steady (or deterministic) wind speed is defined by means of (1) a Weibull distribution curve at a reference elevation of 9.1 meters, from site data, (2) a power-law model of wind shear, in which the exponent is a function of both surface roughness and steady wind speed at the reference elevation, and (3) a corrected Weibull distribution at a selected elevation such as that of the rotor axis in a horizontal-axis machine.

Next, wind speed variations with periods less than approximately 10 minutes are modeled as a Gaussian random process. This random process (wind turbulence) is then characterized by a turbulence spectrum and its associated turbulence intensity. The turbulence spectrum may be narrow or broad - band, depending on the known or assumed response of the wind turbine. For example, wind turbine control systems may eliminate the effects of very slow wind speed variations. At each steady wind speed, the Gaussian random process is then idealized further as a set of discrete gusts having random Gaussian amplitudes but with specified (i.e., deterministic) shapes and periods.

The effects on a wind turbine of these discrete gusts (each of which has an associated probability of occurrence) are predicted by means of deterministic aero-structural dynamic models. Thus, this procedure combines the statistical description of the wind with the physical (deterministic) model of the wind turbine to produce a statistical description of the wind turbine response.

\section{ANALYSIS}

Steady Wind Model

The annual distribution of steady wind speeds is assumed to be given by the following Weibull distribution (Ref. 4): 


$$
p\left(\bar{w} \geq \bar{w}_{p}\right)_{\text {ref }}=\exp \left[-\left(\bar{w}_{p} / c\right)^{k}\right]_{\text {ref }}
$$

in which

$$
\begin{aligned}
\mathrm{p}\left(\bar{W}_{\mathbb{W}} \geq \bar{W}_{\mathrm{p}}\right) & =\text { probability that } \bar{W} \geq \bar{W}_{\mathrm{p}} \\
\bar{W} & =\text { steady wind speed, } \mathrm{m} / \mathrm{s} \\
\bar{W}_{p} & =\text { prescribed value of } \bar{W}, \mathrm{~m} / \mathrm{s} \\
c, k & =\text { empirical curve-fit constants, } \mathrm{m} / \mathrm{s} \text { and } \\
& \text { dimensionless, respectively } \\
\text { ref } & =\text { reference elevation of } 9.1 \mathrm{~m} .
\end{aligned}
$$

The empirical constants $c$ and $k$ in Equation (1) are determined by curvefitting wind speed data taken at or adjusted to the reference elevation of 9.1 meters.

Next, wind speeds at elevations other than the reference elevation are determined by means of the following power-law relationship (Refs. 4 and 5):

$$
\bar{w}=\bar{w}_{\text {ref }}\left(h^{\prime} h_{\text {ref }}\right)^{\alpha} \quad .
$$

As described in Ref. 6 , the exponent $\alpha$ in Equation (2a) is given as a function of both surface roughness and wind speed:

$$
\alpha=a_{0}\left(1-\frac{\log \bar{W}_{r e f}}{\log W_{0}}\right)
$$

where $\alpha_{0}$ is given by:

$$
\alpha_{0}=\left(\frac{z_{0}}{h_{\text {ref }}}\right)^{0.20} .
$$


A comparison of the measured wind shear as well as the specific values of for various wind turbine sites is given in Ref. 7 , in which



Equations (2) are used for two purposes: (1) to adjust the annual distribution curve in Equation (1) from the reference elevation to rotor shaft elevation in order to calculate annual energy production, and (2) to calculate cyclic loads caused by steady wind shear. The surface roughness constant $z_{0}$ is determined by site characteristics (Ref. 5). The relationship (2b) enables the analyst to account for observed variations in wind shear with wind speed (Refs. 5, 7 and 8). Generally, the exponent $\alpha$ decreases with increasing wind speed, becoming negligible at high wind speeds.

Wind Turbulence Model

The wind turbulence associated with a specific steady wind speed at the reference elevation $h_{\text {ref }}$ is assumed to be a Gaussian random process with the following turbulence intensity (Ref. 9):

$$
\begin{gathered}
\sigma_{\text {ref }}=\frac{1.04 \bar{w}_{\text {ref }}}{\operatorname{Ln}\left(h_{\text {ref }} / z_{0}+1\right)} \\
\sigma_{\text {ref }, y}=0.8 \sigma_{\text {ref }, x} \\
\sigma_{\text {ref }, z}=0.5 \sigma_{\text {ref }, x}
\end{gathered}
$$

in which

$$
\begin{aligned}
\sigma_{\text {ref }}= & \text { turbulence intensity (i.e., standard deviation of turbulent } \\
& \text { fluctuations) associated with } \bar{W}_{\text {ref }}, \mathrm{m} / \mathrm{s}
\end{aligned}
$$




$$
\begin{aligned}
x, y, z= & \begin{array}{l}
\text { longitudinal, lateral, and vertical directions, } \\
\text { respectively. }
\end{array}
\end{aligned}
$$

The logarithmic spectrum for the longitudinal component of wind turbulence at a given elevation $h$ is as follows (Ref. 9):

$$
\phi_{x}(n, h, \bar{W})=\frac{\sigma_{r e f, x}^{2}}{n} \frac{0.164\left(n / n_{0, x}\right)}{1+0.164\left(n / n_{0, x}\right)^{5 / 3}}
$$

and

$$
n=\frac{n h}{\bar{W}}
$$

in which

$$
\begin{aligned}
& \phi_{x}=\begin{array}{l}
\text { annual average spectrum for longitudinal component of } \\
\text { wind turbulence, } \mathrm{m}^{2} / \mathrm{s}
\end{array} \\
& n=\text { reduced frequency, dimensionless } \\
& n \quad=\text { annual average circular frequency, } \mathrm{Hz} \\
& n_{0, x}=\text { dimensionless constant, } 0.0144 .
\end{aligned}
$$

Similar equations apply to the lateral and vertical components of wind turbulence, with $n_{0, y}$ equal to 0.0265 and $n_{0, z}$ equal to 0.0962 .

The turbulence intensity at a given elevation $h$ and steady wind speed $W$ can now be calculated for a specific wind turbine by means of the following equation:

$$
\sigma_{x}=\left[\int_{n_{\min }}^{n_{\max }} \phi_{x} d n\right]^{1 / 2}
$$

in which the circular frequency limits, $n_{\max }$ and $n_{\min }$, are obtained from known or assumed response characteristics of the specific wind turbine. 
These characteristics might include control system response rates, gust alleviation devices such as teetering, etc.

Equations similar to (5) are used to calculate lateral and vertical turbulence intensities, $\sigma_{y}$ and $\sigma_{z}$, respectively. The following equations apply to al1 three components, so the subscripts $x, y, z$ are omitted.

The Gaussian random process defined by Equations (4) and (5) is now idealized further as follows: The turbulence of the wind is assumed to consist of a set of discrete gusts with Gaussian random amplitudes but with specified (deterministic) shapes and periods. The assumed shape of each discrete gust is as follows:

$$
W(t)=\bar{W}+A\left[1-\cos \left(\frac{2 \pi t}{T}\right)\right], \quad 0 \leq t \leq T
$$

in which

$$
\begin{aligned}
& t=\text { time, } \mathrm{s} \\
& A=\text { gust amplitude, } \mathrm{m} / \mathrm{s} \\
& T=\text { gust period, } \mathrm{s} .
\end{aligned}
$$

The Gaussian random amplitude A with its turbulence intensity $\sigma$ has the following normal distribution:

$$
p(A)=\frac{1}{\sqrt{2 \pi} \sigma} \exp \left[-\frac{1}{2}\left(\frac{A}{\sigma}\right)^{2}\right]
$$

in which

$$
p(A)=\begin{aligned}
& \text { probability density that a gust will occur with amplitude } A, \\
& \mathrm{~s} / \mathrm{m} \text {. }
\end{aligned}
$$

To complete this definition of the set of discrete gusts, the period $T$ is selected according to the following criteria: Let $T_{0}(A)$ be the most probable period of a gust with amplitude A. In Reference 10, for example, equations are proposed which relate gust period and gust amplitude. Then 


$$
T=\left\{\begin{array}{l}
T_{0} / 2,2 n_{\min } \leq \frac{1}{T_{0}} \leq 2 n_{\max } \\
1 / 2 n_{\min }, \frac{1}{T_{0}}<2 n_{\min } \\
1 / 2 n_{\max }, \frac{1}{T_{0}}>2 n_{\max }
\end{array} .\right.
$$

The effects of these discrete gusts, on a given wind turbine, are then predicted by means of a deterministic aero-structural dynamic model. In this way a statistical description of the wind turbine loads is obtained from the statistical description of the wind.

\section{CONCLUDING REMARKS}

The equations presented herein are a realistic representation of the steady and turbulent nature of the wind environment from the viewpoint of the wind turbine designer. They include the deterministic and stochastic processes which are important to the calculation of the performance and structural integrity of wind machines. However, validation of this gust model by means of wind turbine data is required. 


\section{REFERENCES}

1. Spera, D. A.: Comparison of Computer Codes for Calculating Dynamic Loads in Wind Turbines. DOE/NASA/1028-78/16, NASA TM-73773, 1977.

2. Spera, D. A.; Janetzke, D. C.; and Richards, T. R.: Dynamic Blade Loading in the ERDA/NASA $100 \mathrm{~kW}$ and $200 \mathrm{~kW}$ Wind Turbines. ERDA/NASA/ 1004-77/2, NASA TM-73711, 1977.

3. Frost, W.; and Long, B. H.: Engineering Handbook on the Atmospheric Environmental Guidelines for Use in Wind Turbine Generator Development. NASA TM (to be published).

4. ibid: Chapter 2 .

5. ibid: Chapter 3.

6. Spera, D. A.; Richards, T. R.: A Revised Wind Shear Power Law Model. NASA PIR \#70.

7. Richards, T. R.; Spera, D. A.: A Comparison of Measured Wind Shear at Various Wind Turbine Sites. NASA PIR \#73, Sept. 29, 1977.

8. Justus, C. G.; Hargraves, W. R.; and Mikhail, A.: Reference Wind Speed Distributions and Height Profiles for Wind Turbine Design and Performance Evaluation Applications. ORO/5108-76/4 UC 60, 1976.

9. Frost, W.; and Long, B. H.: 10c. cit, Chapter 4.

10. Terrestrial Environment (Climatic) Criteria Guidelines for Use in Aerospace Vehicle Development, 1977 Revision. John W. Kaufman, Ed. NASA TM-78118, 1977. 
APPENDIX B

PRELIMINARY DEMONSTRATION OF A DISCRETE GUST MODEL INVOLVING INTRINSIC GUST AMPLITUDE AND INTRINSIC GUST TIME 
APPENDIX B

PRELIMINARY DEMONSTRATION OF A DISCRETE GUST MODEL INVOLVING INTRINS IC GUST AMPLITUDE AND INTRINSIC GUST TIME

In Section 2 a general definition of discrete gust was followed by two examples called GUST 0 and GUST in $_{1}$ which a turbulence time series was divided into a series of discrete events, each with an intrinsic measure of gust amplitude and gust time. GUST 0 and GUST, were illustrated in Figure 2.1. The conceptual framework of a model was presented in Equations (2.1) through (2.3). In Section 4 it was shown how each of the models reviewed relates to part of the concept of these equations, but none of them relate to all of it. In this Appendix we present analysis of a single 55-minute test period at one anemometer as a preliminary example of the broader discrete gust concept entailed in Equation (2.1). A more complete development is underway at PNL, which will treat several aspects not considered here such as conditional probability of either amplitude or time when the other is fixed, and the use of a rotating frame of reference.

The actual analysis is preceded by a description of the data and site, analytical specification of the digital filters, and a few comments relating horizontal wind speed to the turbulence $u$-component.

This analysis applies primarily to GUST, events. Unfortunately a GUST, analysis is very sensitive to an arbitrary specification that has no analog in the GUSTo analysis. This is a tolerance, the minimum velocity change that will be defined as a GUST, event. Tolerance is illustrated in Figure B.1, in which an arbitrarily chosen tolerance is shown along with inflections in the time series that qualify as discrete gusts under this definition and one that does not. The arbitrary tolerance interval is shown at the left margin. The small inflection on the left side of the function is too small in amplitude to qualify as a GUST, event. On the other hand, if the tolerance were made small enough that this inflection could qualify, 


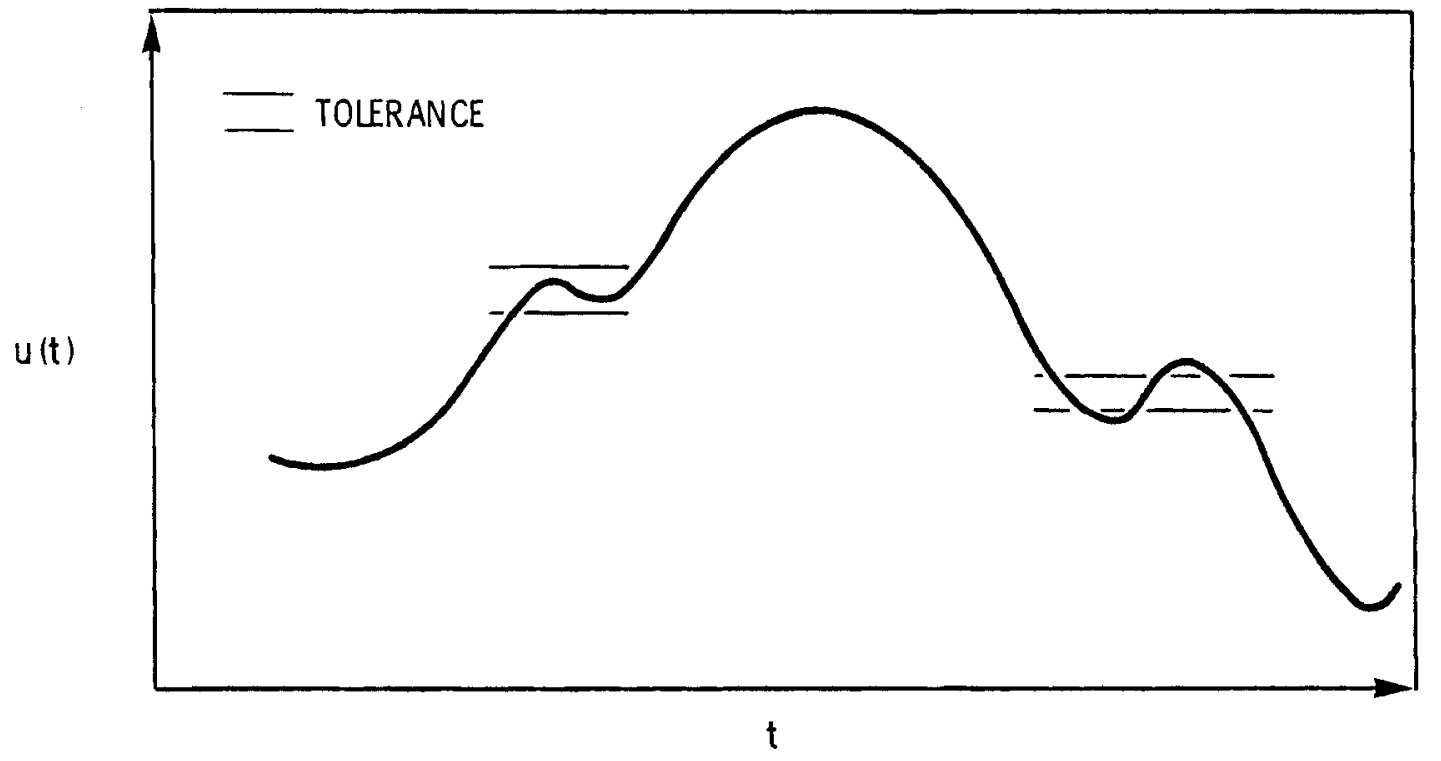

FIGURE B.1. Velocity Fluctuations Relative to GUST, Tolerance

the first GUST ${ }_{1}$ event in this figure would be divided into three GUST events, $_{1}$ and the statistics accordingly changed.

The analysis includes marginal probabilities of GUST 0 amplitude and time, the frequency of zero crossings, and a table of moments.

\section{B. 1 EXPER IMENTAL DATA}

The experimental data analyzed in this document were taken from a Gill U-V-W propeller anemometer located at a height of $36 \mathrm{~m}$ over level desert terrain at the Hanford meteorological site. The vegetation consists of widely scattered sagebrush plants about a meter high and clumps of desert grasses, at a location where the roughness length $z_{0}$ is estimated at $5 \mathrm{~cm}$.

The data constitute a 55-minute segment starting at 2000 local time on July 31, 1978. The original digital data are at a sampling interval of $0.1 \mathrm{sec}$. The data analyzed were block averaged four at a time to yield discrete data at an interval of $0.4 \mathrm{sec}$. 
The mean wind speed at the height of measurement was $8.7 \mathrm{~m} \mathrm{sec}^{-1}$. From simultaneous measurement of wind speed and temperature at several levels of the nearby Hanford $125-\mathrm{m}$ tower, a value of 100 to $150 \mathrm{~m}$ is estimated for the Monin-Obukhov stability length. In order words, the density stratification at the height of measurement was slightly stable.

\section{B. 2 DIGITAL FILTERING}

The bandpass wind fluctuation filtering properties of a WECS machine were simulated by applying digital filters that are moving averages to the data. The formulation is as follows: Let the time series consist of $N$ values at a sampling interval $\Delta t$;

$$
u(0), u(\Delta t), u(2 \Delta t), \ldots \ldots u[(N-1) \Delta t]
$$

The filtering technique consists of using moving averages with the length adjusted so that the half-power point of the filter is the required frequency. Let $S_{1}$ be the number of data required in a moving average that will filter the data such that the half power point of a lowpass filter is at $s_{1}$ seconds. Let $S_{2}$ be the number of data required in a moving average to be used to produce highpass filtered data with half power point at $s_{2}$ seconds. Then $S_{1}$ and $S_{2}$ must be optimal odd integers approximating

$$
\begin{aligned}
& s_{1}=0.44 \mathrm{~s}_{1} / \Delta t \\
& S_{2}=0.76 \mathrm{~s}_{2} / \Delta t
\end{aligned}
$$

We define the moving average filter centered at time mat by

$$
\psi\left[u(m \Delta t), s_{i}\right]=\frac{1}{S_{i}} \sum_{j=m-\frac{S_{i}-1}{2}}^{m+\frac{S_{i}-1}{2}}[u(j \Delta t)], \frac{S_{i}-1}{2} \leq m \leq(N-1)-\frac{S_{i}-1}{2}
$$


depending on whether the moving average is to be used in a lowpass or highpass filter. The data $u(m \Delta t)$ as seen through the lowpass filter or the highpass filter are, respectively,

$$
\begin{gathered}
u_{L}\left(m \Delta t, S_{1}\right)=\psi\left[u\left(m \Delta t, S_{1}\right)\right] \\
u_{H}\left(m \Delta t, S_{2}\right)=u(m \Delta t)-\psi\left[u\left(m \Delta t, S_{2}\right)\right] .
\end{gathered}
$$

A bandpass filter using both characteristics is given by

$$
u_{B}\left(m \Delta t, S_{1}, S_{2}\right)=\psi\left[u_{H}\left(m \Delta t, S_{2}\right), S_{1}\right] .
$$

In other words, the bandpassed data are run first through the highpass filter and then through the lowpass filter.

The corresponding multipliers applied to the spectral estimates of the unfiltered data, which yield the spectra of the filtered data, are:

$$
\phi_{L}\left(n, S_{1}\right)=\phi(n)\left[\frac{\sin \left(S_{1} \pi n \Delta t\right)}{S_{1} \sin (\pi n \Delta t)}\right]^{2}
$$

for the lowpass filter, and

$$
\phi_{H}\left(n, S_{2}\right)=\phi(n)\left[1-\frac{\sin \left(S_{2} \pi n \Delta t\right)}{S_{2} \sin (\pi n \Delta t)}\right]^{2}
$$

for the highpass filter. The expressions in the brackets are the spectral transfer functions. For the bandpass filter, $\phi(n)$ is multiplied by both bracketed squared functions.

Besides presenting data that have not been digitally filtered, this appendix presents analysis of data subjected to six different filterings. We regard the basic filter as a bandpass filter consisting of a $50-\mathrm{sec}$ 
highpass filter and a 5-sec lowpass filter. The others are derived from it by taking either the highpass or the lowpass part or by moving the bandpass filter up or down on octave. These filters and the notations we employ are:

$\begin{array}{rl}0 & \text { no digital filter } \\ 50 /- & 50 \text { second highpass filter } \\ -/ 5 & 5 \text { second lowpass filter } \\ 100 / 10 & 100 \text { to } 10 \text { second bandpass filter } \\ 50 / 5 & 50 \text { to } 5 \text { second bandpass filter } \\ 25 / 2.5 & 25 \text { to } 2.5 \text { second bandpass filter. }\end{array}$

\section{B.3 COMMENTS ON USE OF SPEED AND ANGLE RATHER THAN HORIZONTAL WIND COMPONENTS}

Since wind turbine generators are oriented to face the mean wind according to some kind of strategy, it is felt that horizontal wind speed and wind angle are more representative of the wind as seen by the machine than are the wind longitudinal and lateral components $U$ and $V$. Accordingly, we have defined:

$$
\begin{aligned}
& \text { horizontal wind speed }(t)=\sqrt{U^{2}(t)+V^{2}(t)} \\
& \text { horizontal wind angle }(t)=\operatorname{Tan}^{-1}[V(t) / U(t)] .
\end{aligned}
$$

For this data analysis we find no significant differences between analysis of speed and angle versus analys is of the components. Therefore, we have used the $u$ subscript for either u-component or speed analysis. Cross analysis of $U$ with speed or of $V$ with angle shows a normalized cross covariance of 0.98 in either case. The power spectra are of essentially the same shape. The speed variance is $3 \%$ greater than the $U$ variance. Also, the average value of the speed is $2 \%$ greater than $\bar{U}$. The horizontal angle variance is within $0.5 \%$ of that obtained by dividing the $V$-component variance by the square of the mean horizontal speed. For these reasons we consider analysis of $U$ and speed as interchangeable, and similarly with $V$ and angle. In this report the angle analysis is not exhibited. 


\section{B.4 PROBABILITY ANALYSIS}

In this analysis we deal with positive quantities: the gust time and the absolute value of gust amplitude. Inspection of the histogram boxes of both time and amplitude shows maximum probability density either in the lowest boxes (those adjacent the zero value) or in a neighboring box. Departures from this pattern are particularly minimized for GUST filtered data, which are our primary interest. Therefore, we shall assume that the maximum probability density is at the zero argument and accordingly characterize the probability distribution with non-central moments. We now define the parameters in Equation (2.1a). The $E$ notation below is the expectation operator. We define:

$$
\begin{aligned}
& \bar{A}=E[|A|] \\
& \bar{T}=E[T] \\
& \sigma_{A}=\sqrt{E\left[A^{2}\right]} \\
& \sigma_{T}=\sqrt{E\left[T^{2}\right]} \\
& \rho_{A T}=\frac{E\left[(|A|-\bar{A})(T-\bar{T})^{2}\right]}{E\left[(A-\bar{A})^{2}\right] E\left[(T-\bar{T})^{2}\right]}
\end{aligned}
$$

We also define kurtosis values for turbulence, gust amplitude, and gust time by $K_{U}, K_{A}$, and $K_{T}$, respectively:

$$
\begin{aligned}
& K_{u}=\frac{E\left[u^{4}\right]}{\left(\sigma_{u}^{2}\right)^{2}} \\
& K_{A}=\frac{E\left[A^{4}\right]}{\left(\sigma_{A}^{2}\right)^{2}}
\end{aligned}
$$




$$
K_{T}=\frac{E\left[T^{4}\right]}{\left(\sigma_{T}^{2}\right)^{2}} \text {. }
$$

The probability distributions, the amplitudes and times of GUST 0 events are shown in Figures $B .2$ and $B .3$ for unfiltered data and data filtered with the 50/5-sec bandpass filter, respectively. In these figures the symmetrical properties of the Gaussian scales are used to plot the probability distributions of two related functions that take on positive values. The choice of scales implies that the first probability distribution we wish to test for fit is the half-Gaussian.

The probability distribution of amplitude appears on the right half of the graph, while that of the corresponding times is shown on the left half of the graph. The probability distribution scale at the bottom of the left half of the graph increases from right to left. The x-axis scaling on each half of the graph is such that if the probability distribution is halfGaussian, the sample points will form a straight line; the slope of this line is proportional to the rms of the data. In the center of the graph, the correlation coefficient and the basic frequency factor (which is extrinsic to the discussion in Section B.4) are given. The kurtosis of amplitudes and times is given in the lower right and left corners of the graph.

The dots on the graph indicate the probability distributions of GUSTO amplitude and time found from analys is of the aforementioned data. These are the sample distributions.

Our interpretation of Figures B.2 and B.3 is that the Gaussian model is inadequate for unfiltered data, but tentatively adequate for the bandpass filtered data -- tentatively because there is no theoretical backup and because the data sample is so small. This is better than if the Gaussian model were adequate for unfiltered data and inadequate for bandpass filtered data, since we are principally interested in the attempt to model the fluctuations as the machine sees them. Since the probability of GUST 0 times for unfiltered data has a kurtosis of 18 , the Gaussian model is certainly 


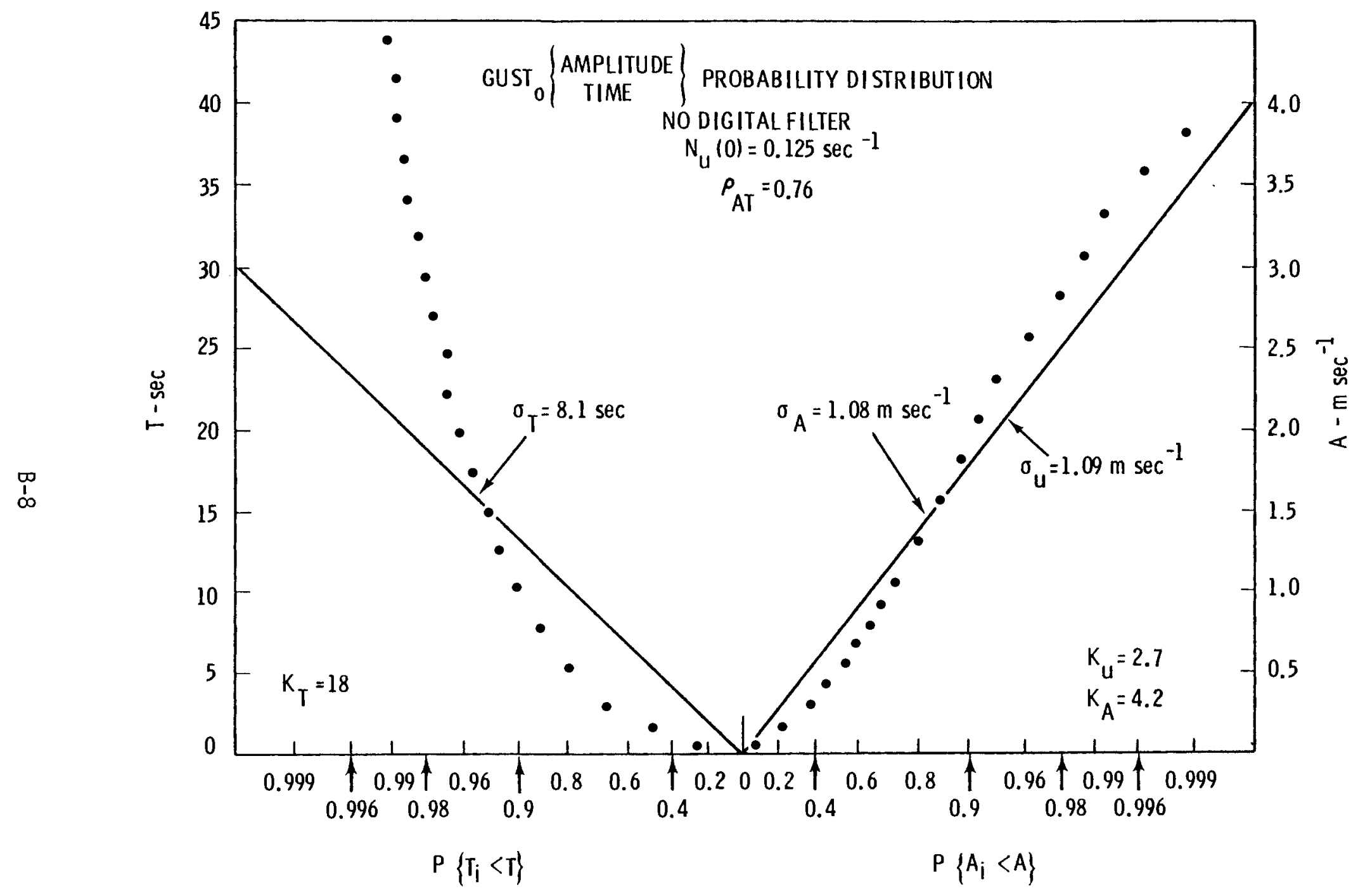

FIGURE B.2. Statistics for GUST 0 Speed - No Filter 


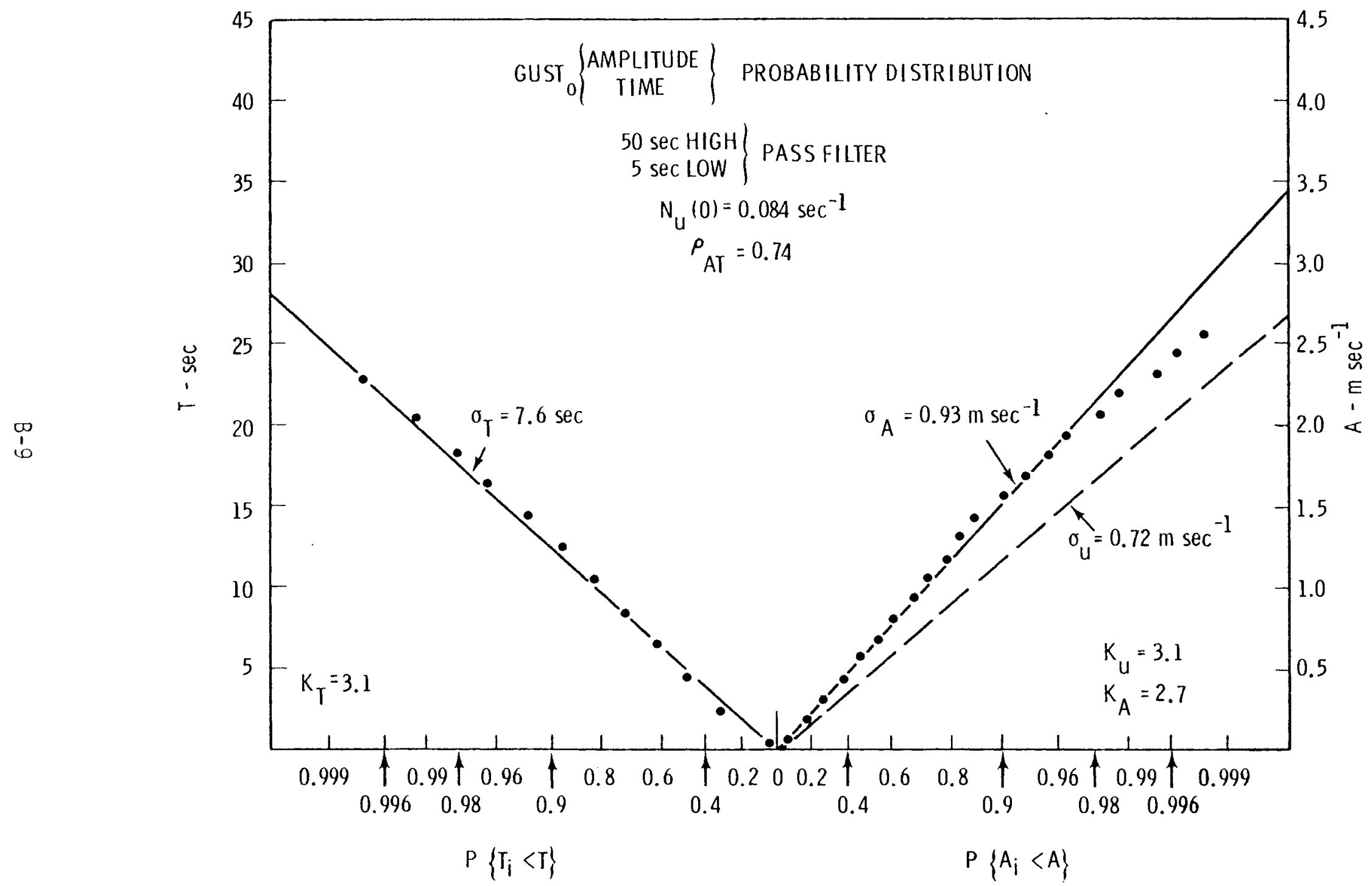

FIGURE B.3. Statistics for GUST 0 Speed - 50/5 Bandpass Filter 
not adequate. The graph and the kurtosis of 4.2 for the amplitudes show that the sample distribution systematically and significantly deviates from the Gaussian. Here a reference should be made back to Figure $4.2 a$, where in another graphic projection the same sample data are compared to the Gaussian and an exponential model. Neither fit is good.

In the case of the data filtered with the $50 / 5$ bandpass filter, the times definitely follow Gaussian probability, and the amplitudes less definitely so. Here Gaussian probability is a conservative estimator at large amplitudes. However, we will accept this approximation as reasonable, and note also the high correlation (not the correlation squared) between amplitude and time.

The high kurtosis for GUST, amplitudes and times in unfiltered data may be explained as follows. It has been known for some time that the kurtosis of turbulence is higher than the Gaussian 3.0 (Batchelor 1953). However, from Table B.1 we see that the turbulence kurtosis for the unfiltered data was 2.7, while that for the $50 / 5$ bandpass filtered data was 3.1. The lower kurtosis for the unfiltered data is caused by the presence of low-frequency quasi-two-dimensional meanders imposed upon the three-dimensional turbulence. The contribution of the meanders lowers the turbulence kurtosis because the change tends toward sinusoidal distribution, which has low kurtosis. However, one of these long waves may define only one GUST 0 event. If so, they make one outstanding contribution to GUST, amplitude statistics and one even more outstanding contribution to GUST $_{1}$ statistics. Therefore, the GUST 0 statistics for such data have a few outstanding outliers and thus have high kurtosis.

\section{B.5 GUST FREQUENCY}

The frequency of GUST 0 events is the frequency with which the turbulence function crosses its own mean value. This is twice the frequency of positive-slope zero crossings as described and theoretically developed by Rice (1945). (See Equation (4.2) and Appendix C.) 
TABLE B.1. GUST Parameters and Ratios Among Scale Parameters from Analysis of One Test $(a)$

\begin{tabular}{|c|c|c|c|c|c|c|c|}
\hline & $\begin{array}{l}\text { Filter } \\
\text { (sec) } \\
\end{array}$ & 0 & $50 /-$ & -15 & $\underline{100 / 10}$ & $50 / 5$ & $25 / 2.5$ \\
\hline$o_{u}$ & $\mathrm{~m} \mathrm{sec}^{-1}$ & 1.09 & 0.80 & 1.04 & 0.75 & 0.72 & 0.61 \\
\hline$N_{u}(0)$ & $\sec ^{-1}$ & 0.125 & 0.181 & 0.054 & 0.045 & 0.084 & 0.142 \\
\hline$\sigma_{A}$ & $\mathrm{~m} \mathrm{sec}^{-1}$ & 1.08 & 0.92 & 1.19 & 0.98 & 0.93 & 0.79 \\
\hline${ }^{\sigma_{T}}$ & sec & 8.1 & 4.2 & 15.0 & 14.7 & 7.6 & 4.4 \\
\hline $\mathrm{k}_{\mathrm{u}}$ & & 2.7 & 3.2 & 2.6 & 3.0 & 3.1 & 3.4 \\
\hline$k_{A}$ & & 4.2 & 3.6 & 3.0 & 2.6 & 2.7 & 2.9 \\
\hline $\mathrm{k}_{\mathrm{T}}$ & & 18.0 & 6.4 & 9.0 & 3.9 & 3.1 & 2.9 \\
\hline$\sigma_{A} / \sigma$ & & 0.99 & 1.15 & 1.14 & 1.31 & 1.29 & 1.29 \\
\hline${ }^{20} T_{T} N(0)$ & & 2.02 & 1.52 & 1.63 & 1.32 & 1.28 & 1.25 \\
\hline $100 \sigma_{\mathrm{A}} / \mathrm{U \sigma}_{\mathrm{T}}$ & & 1.53 & 2.52 & 0.91 & 0.77 & 1.41 & 2.06 \\
\hline$\rho_{A T}$ & & 0.76 & 0.77 & 0.76 & 0.71 & 0.74 & 0.68 \\
\hline
\end{tabular}




For filtered turbulence with spectrum $\phi(n) F(n)$, Rice's expression can be written as in Equation (4.2). To avoid confusion we will adopt his meaning although twice that figure would be more appropriate for our usage.

Since a theoretical base exists, we compare the results of our data analysis with Rice's frequency. The comparison is shown in Figure B.4. Here the frequency, computed by simply counting through the time series and shown on the graph by the solid dots, is compared to the frequencies calculated by direct application of Rice's equation with the model spectrum of Equations (3.12) through (3.14) shown by the open circles. If we discount the estimates for data not bandpass filtered, i.e., the first three on the graph, we see that all the $\mathrm{N}_{u}(0)$ values estimated using the spectral technique are easily within $20 \%$ of the values found by counting through the data. On the other hand, the estimates for data not subjected to the bandpass filter are off by as much as $40 \%$.

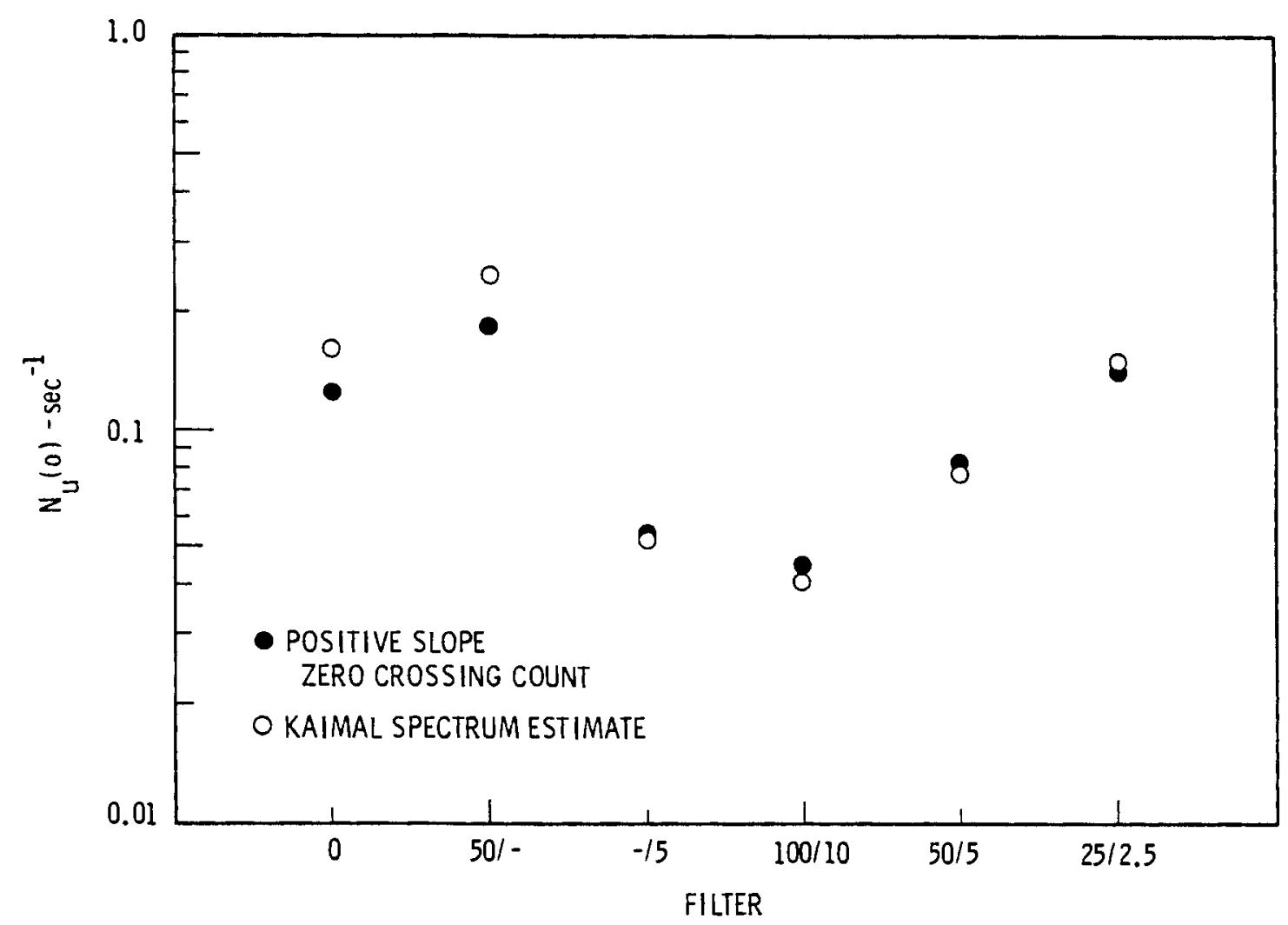

FIGURE B.4. Statistics for GUST 0 Speed With 50/5 Second Bandpass Filter 
The fact that one of these bad fit cases involves data that are highpass filtered and thus suitable for GUST, analysis is disturbing and may occur because the particular highpass filter used not only filters but inflates spectral estimates by more than $40 \%$ of certain frequencies, as can be seen by maximizing the value of the transfer function on the right hand factor in Equation (B.8). Therefore, the goodness of fit in the three bandpass cases may be fortuitous.

Until more case studies have been completed, we observe that the present example is a favorable indication regarding the use of the spectral technique of Appendix C and Equations (3.12) through (3.14) for estimating the GUSTO basic frequency factor, the frequency of positive-slope zero-level crossings. Also, we note that the data were taken over a relatively homogeneous site.

\section{B. 6 CHARACTERISTIC MAGNITUDE ANALYSIS}

In order to make a useful model of Equations (2.1) through (2.3), the gust parameters $\sigma_{A}, \sigma_{T}$ and $\rho_{A T}$ must be characterized. Equations (2.1b) through (2.1d) gave a rather abstract indication of how this may be done. Here we set forth some tentative results related to the ideas of these equations. We observe that the basic frequency factor is half the inverse of the average gust time. We can write

$$
\bar{T}=0.5 / N_{u}(0)
$$

for GUST $_{0}$. Therefore, the relation of $\sigma_{T}$ to $N_{U}(0)$ involves only the probability distribution of the GUST, times. For a Gaussian process, the ratio between rms and mean value is $\sqrt{\pi / 2}$, or about 1.25 .

We can also predict a relation between $\sigma_{A}$ for GUST and the turbulence. Consider the case where the width of the bandpass filter approaches zero: The turbulence signal approaches the form of a pure sine wave. If the ampiitude is $A, \sigma_{A}=A$ and $\sigma_{U}$ is $A / \sqrt{2}$. Therefore, the ratio $\sigma_{A} / \sigma_{U}$ approaches 1.41 as the bandwidth approaches zero. In Table B.1 these ratios are exhibited for the different filterings. 
The ratios $\sigma_{A} / \sigma_{U}$ and $\sigma_{T} / \bar{T}$ exhibit the expected behavior, and tentatively we observe that for narrowly bandpassed data, these ratios are both about 1.3. The correlation coefficient is uniformly high. The correlation coefficient and the associated joint probability distribution of amplitude

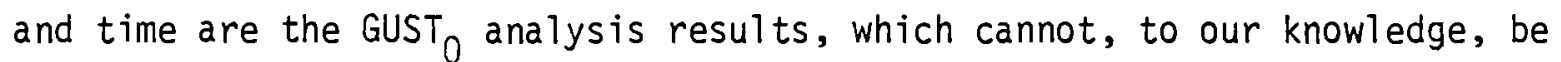
derived theoretically from conventional turbulence analysis.

In Figure B.5 the various rms for turbulence GUST $_{0}$ and GUST 1 events are shown along with kurtosis of the same distributions. This figure emphasizes that gust times are much more sensitive to filtering than are the gust amplitudes. This is a logical result for narrow bandpass filtering, since as the bandwidth approaches zero, the gust time is the inverse of the frequency of the filter. The fact that $\sigma_{T}$ changes almost by a factor of two as the filter is moved up or down an octave shows that for the decade band the apparent gust time has little to do with a gust time scale based on atmospheric behavior alone.

Also from Figure B.5 and Table B. 1 we observe that for filtered data the GUST $_{0}$ rms is greater than the turbulence rms.

This preliminary analysis indicates that, for filtered data, there is much hope that analysis of GUST 0 discrete events can be easily related to conventional turbulence analysis. 

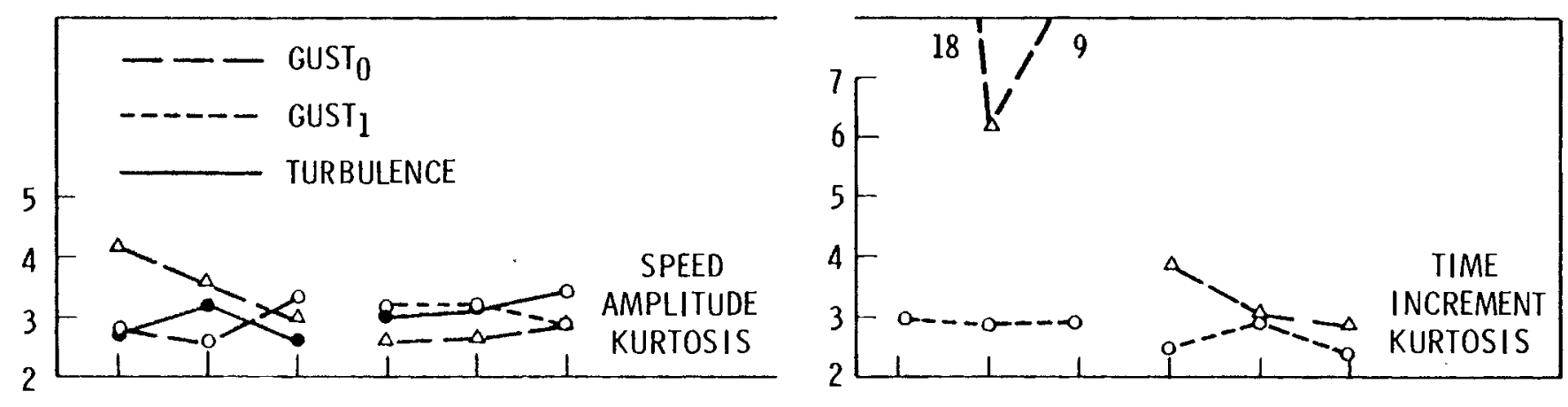

$\frac{p}{1}$
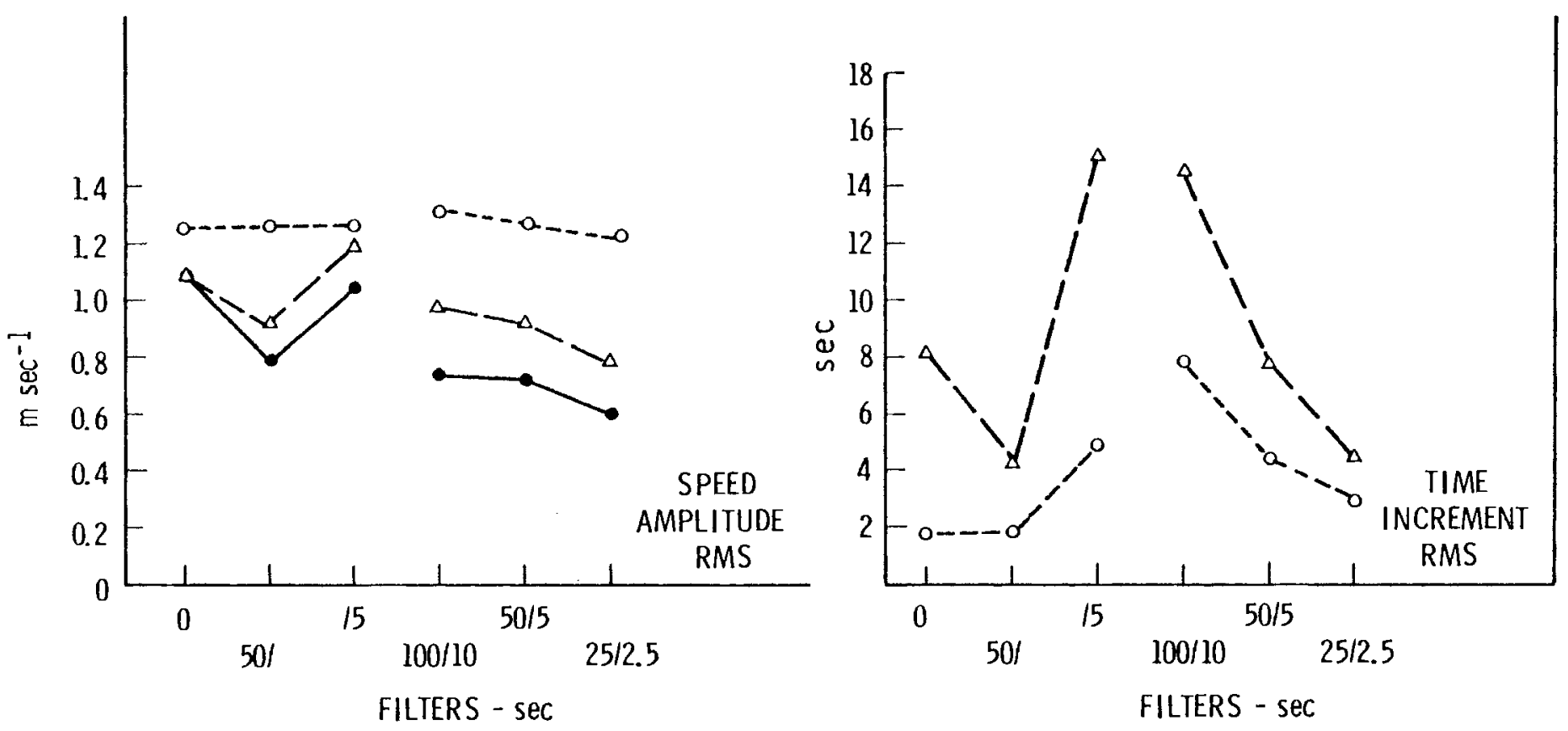

FIGURE B.5. Characteristic Magnitude Analysis for Horizontal Wind Speed 


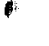

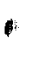




\section{APPENDIX C}

CALCULATION OF FREQUENCY OF ZERO-CROSSINGS FROM TURBULENCE SPECTRA 


\section{CALCULATION OF FREQUENCY OF ZERO-CROSSINGS}

FROM TURBULENCE SPECTRA

In principle the frequency of positive-slope zero crossings can be calculated as the square root of the quotient of two spectral integrals. In practice it is also necessary to supply some lowpass filter, or cutoff frequency, since the integral in the numerator does not converge when a standard turbulence spectrum is used. In practice there is also considerable uncertainty inherent in the specification of a length scale, which is unavoidable when any of the standard forms of a dimensionless spectrum are adapted. Therefore, little more is lost if the filter $F(n)$ consists simply of a pair of arbitrary cutoff frequencies of integration, i.e.,

$$
\begin{array}{ll}
F(n)=1 & n_{1} \leq n \leq n_{2} \\
F(n)=0 & \text { otherwise } .
\end{array}
$$

Using this filter and remembering that standard atmospheric spectra are of the form $n \phi_{u}(n)$ rather than $\phi_{u}(n)$, we rewrite Equation (4.4) as

$$
N_{u}\left(0 ; U, n_{2}: n_{1}\right)=\left[\frac{\int_{n_{1}}^{n_{2}} n^{2} \psi_{u}(n) d[\operatorname{Ln}(n)]}{\int_{n_{1}}^{n_{2}} \psi_{u}(n) d[\operatorname{Ln}(n)]}\right]^{1 / 2}
$$

where

$$
\psi(n)=n \phi(n)
$$


can be any spectrum of this form, e.g., see Equations (3.12) through (3.14).

The necessary summation can easily be programmed for a hand calculator. When Equations (3.12) through (3.14) are inserted into Equation (C.2), the result is

$$
\begin{gathered}
N_{u}\left(0 ; U, n_{2}: n_{1}\right)=\left[\frac{\sum_{n=n_{1}}^{n_{2}} \frac{n^{2}\left(f / f_{\text {ou }}\right)}{1+0.164\left(f / f_{\text {ou }}\right)^{5 / 3}}}{\left.\sum_{n=n_{1}}^{n_{2}} \frac{f / f_{\text {ou }}}{1+0.164\left(f / f_{\text {ou }}\right)^{5 / 3}}\right]^{1 / 2}}\right. \\
f=n z / U \\
f_{\text {ou }}=0.0144
\end{gathered}
$$

where successive values of $n$ in the summation must be logarithmically spaced, i.e.,

$$
\left(n_{i+1} / n_{i}\right)=\text { constant }=k \quad .
$$

This is because the differential, $d \operatorname{Ln}(n)$, in the integration becomes $\Delta L n(n)$ in the summation. This term does not appear in Equation (C.4) because it cancels out. The frequency range $n_{1}$ to $n_{2}$ defines a bandpass filter. Since the result is particularly sensitive to the upper 1 imit of integration, the result will also be sensitive to the ratio in Equation (C.5) if this ratio is too large and if $n_{c}$ in the summation is approximated as the nearest frequency to the product of $n_{1}$ and some power of $k$ in Equation (c.5), i.e., 
if

$$
n_{2}=n_{1} K^{M}
$$

where $M$ is an integer. For this reason, we recommend that

$$
\log _{10} K \leq 0.02
$$




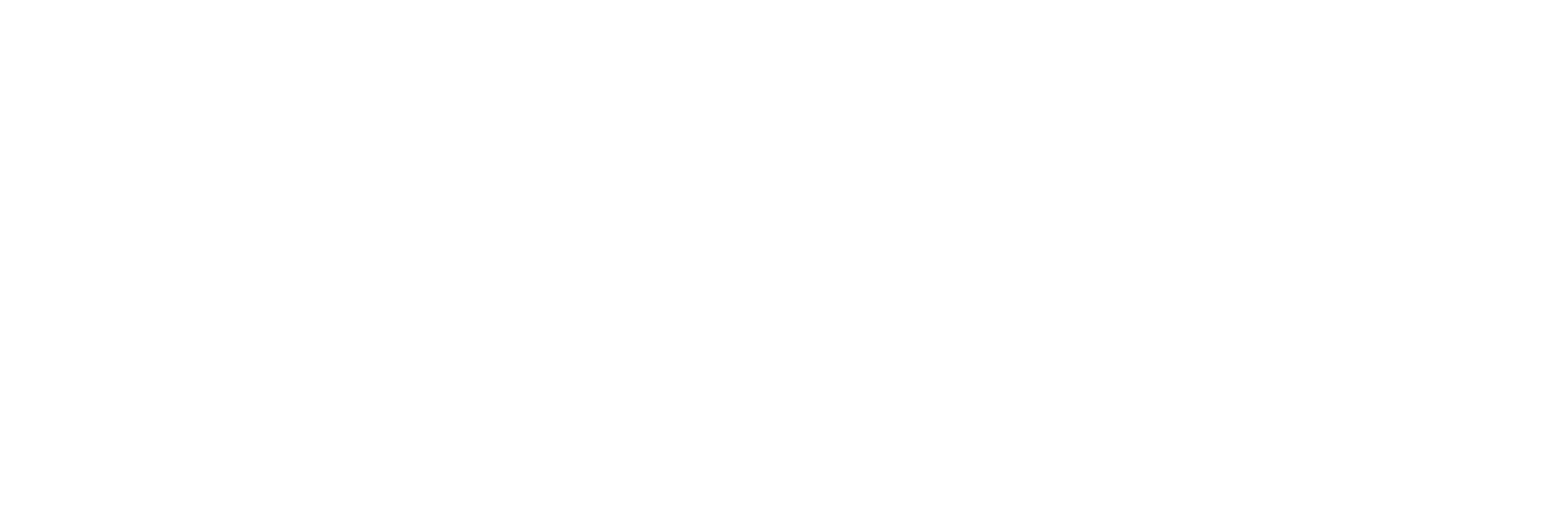




\section{DISTRIBUTION}

No. of

Copies

OFFSITE

A.A. Churm

DOE Chicago Patent Group

9800 S. Cass Avenue

Argonne, IL 60439

C.I. Aspliden

Department of Energy

600 E Street, N.W.

Washington, DC 20545

G.P. Tennyson

Department of Energy

Albuquerque Operations office

P.0. Box 5400

Albuquerque, NM 87115

27 DOE Technical Information Center

W. Frost

FWG Associates, Inc.

271A Lakewood Drive

Tullahoma, TN 37388

G. Fichtl

7703 Oakridge Drive

Huntsville, AL 35802

J.M. Kos

Hamilton Standard Div. of UTC

Bradley Field Road

Windsor Locks, CT 06096

Jean Mayhew

Mail Stop I-M-3

Hamil ton Standard

windsor Locks, CT 06096

M.A. Bowes

Kaman Aerospace Corporation

old Windsor Road

Bloomfield, CT 06095

T.R. Richard

NASA/LERC

21000 Brookpark Road

Cleveland, $\mathrm{OH} 44135$
No. of

Copies

OFFSITE

D. Spera

NASA/LERC

21000 Brookpark Road

Cleveland, $\mathrm{OH} 44135$

J.C. Kaimal

NOAA/ERL/WPL

3000 Marine Street

Boulder, CO 80302

W. Holly

Department of Mechanical Engr.

Oregon State University

Corvallis, OR 97331

J. Dutton

Department of Meteorology

Pennsylvania State University

University Park, PA 16902

H.A. Panofsky

Department of Meteorology

Pennsylvania State University

University Park, PA 16802

A.C. Hansen

Rockwell International

P.0. Box 464

Golden, CO 80401

E.G. Kadlec

Sandia Labs 4715

Box 5800

Albuquerque, NM 87165

R.H. Kurchhoff

Department of Mechanical Engr. University of Massachusetts

Amnerst, MA 01003

A.B. Van Rennes

The Bendix Corporation

Executive Offices

Bendix Center

Southfield, MI 48037 
No. of

Copies

\section{OFFSITE}

F.S. Stoddard

U.S. Windpower Association

25 Adams Street

Burlington, MA 01803

R. Akins

Dept. of Engr. Sci. \& Mech.

Virginia P.I. and S.U.

Blacksburg, VA 24061

\section{ONSITE}

3 DOE Richland Operations Office

P.O. Box 550

Richland, WA 99352

H.E. Ransom

R.K. Stewart

S. Smith

52 Pacific Northwest Laboratory

Battelle Boulevard

Richland, WA 99352

W.C. Cliff

J.R. Connel1

J.C. Doran

R.L. Orake

C.E. Elderkin

C.H. Huang

E.L. Owzarski

W.T. Penne 11

D.C. Powe 11

J.V. Ramsde 11

D.S. Renne

H.L. Wegley

L.L. Wende11

R.K. Woodruff

Technical Information Files (5)

Publishing Coordination (2) 